\title{
Photophysical and Redox Properties of Dinuclear Ru and Os Polypyridyl Complexes with Incorporated Photostable Spiropyran Bridge
}

\author{
Ron T. F. Jukes, ${ }^{\dagger}$ Biljana Bozic, ${ }^{\ddagger}$ Peter Belser, ${ }^{\ddagger}$ Luisa De Cola, ${ }^{,, \S}$ and František Hartt ${ }^{\star, \dagger}$ \\ Van 't Hoff Institute for Molecular Sciences, University of Amsterdam, Nieuwe Achtergracht 166, \\ 1018 WV Amsterdam, The Netherlands, Institute of Inorganic Chemistry, University of Fribourg, \\ Pérolles CH-1700 Fribourg, Switzerland, and Physikalisches Institut, Westfälische \\ Wilhelms-Universität, Mendelstrasse 7, 48149 Münster, Germany
}

\begin{abstract}
Aimed at creating a true photoswitchable energy transfer system, four dinuclear complexes containing ruthenium(II) and osmium(II) metal centers bridged by spiropyran-type linkers were designed and investigated. The bridge in its closed spiropyran form was shown to be a good insulator for energy transfer between the Ru-bpy donor and the Os-bpy acceptor (bpy $=2,2^{\prime}$-bipyridine). On the basis of properties of previously reported photochromic nitrospiropyrans substituted with a single polypyridine metal center, conversion of the bridge to the open merocyanine form was envisaged to result in efficient electronic energy transfer by a sequential ("hopping") mechanism. In contrast to the expectations, however, the studied closed-form dinuclear complexes remained stable independently of their photochemical or electrochemical activation. This difference in reactivity is attributed to the replacement of the nitro group by a second polypyridine metal center. We assume that these changes have fundamentally altered the excited-state and redox properties of the complexes, making the ring-opening pathways unavailable.
\end{abstract}

\section{Introduction}

Much effort has been devoted to the investigation of photochromic compounds, that is, systems existing in two forms that can be interconverted using excitation with light of different wavelengths as a stimulus. ${ }^{1-3}$ The two forms generally differ in their physicochemical properties such as molecular geometry, electronic delocalization, color and reactivity. The interest in these compounds arises from their envisaged application as materials in molecular electronics and data storage ${ }^{4-7}$ first suggested by Hirshberg in $1956 .^{8}$

\footnotetext{
* To whom correspondence should be addressed. E-mail: decola@ uni-muenster.de (L.D.C.), f.hartl@uva.nl (F.H.).

University of Amsterdam.

University of Fribourg.

$\S$ Westfälische Wilhelms-Universität.

(1) Crano, J. C.; Guglielmetti, R. J. Organic Photochromic and Thermochromic Compounds vol. 1: Photochromic Families; Kluwer: Dordrecht, 1999.

(2) Dürr, H.; Bouas-Laurent, H. Photochromism: Molecules and Systems; Elsevier: Amsterdam, 2003.

(3) Feringa, B. L. Molecular Switches; Wiley-VCH: Weinheim, 2001.

(4) Raymo, F. M. Adv. Mater. 2002, 14, 401-414.

(5) Joachim, C.; Gimzewski, J. K.; Aviram, A. Nature 2000, 408, 541548.

(6) Irie, M. E. Chem. Rev. 2000, 100.

(7) Balzani, V.; Credi, A.; Venturi, M. ChemPhysChem 2003, 4, 49-59.

(8) Hirshberg, Y. J. Am. Chem. Soc. 1956, 78, 2304-2312.
}

Photochromic molecules can also be incorporated into larger systems, to acquire functionalities they do not possess by themselves. One interesting possibility is to implement the photochromic molecule as a bridge between an energy donor and acceptor in a system for energy transfer processes. It should then be possible to switch the energy transfer ON and OFF by reversibly converting the bridge.

So far, two donor-bridge-acceptor systems with a photochromic moiety incorporated in the bridge have been reported in the literature. ${ }^{9,10}$ These triads, however, are not true energy transfer switches. The energy levels of the bridge lie in between those of the donor and acceptor termini when the photochromic moiety is in the initial state, thereby facilitating the energy transfer. Then, having converted the bridge photochemically to the other state, the energy levels of the photochromic unit fall below those of the acceptor moiety, so that the excitation energy is ultimately transferred to the bridge. To constitute a true energy transfer modulator, the photochromic molecule should act solely as a switch,

(9) Walz, J.; Ulrich, K.; Port, H.; Wolf, H. C.; Wonner, J.; Effenberger, F. Chem. Phys. Lett. 1993, 213, 321-324.

(10) Jukes, R. T. F.; Adamo, V.; Hartl, F.; Belser, P.; De Cola, L. Coord. Chem. Rev. 2005, 249, 1327-1335. 
Scheme 1. Photoinduced and Thermal Interconversion between Closed (Sp) and Open (Mc) Forms of a Typical Photochromic Spiropyran

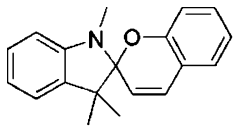

closed form
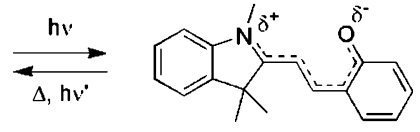

open form influencing the rate of energy transfer between the donor and acceptor, but never functioning as either the donor or the acceptor itself.

Spiropyrans belong to the most intensively investigated classes of photochromic molecules. ${ }^{11-13}$ The conjugation in a spiropyran molecule is broken over the single spiro-carbon atom shared by the two subunits, which strongly limits their mutual electronic interaction. ${ }^{14,15}$ The bond between the spiro-carbon and oxygen atoms in a spiropyran can be broken photochemically, resulting in the formation of a planar merocyanine form stabilized by extensive $\pi$-conjugation over nearly the entire molecule (see Scheme 1). At room temperature, the open form reverts thermally to the closed precursor.

In a preceding publication, we described the properties of bpy-substituted nitrospiropyran 3c, its mononuclear $\mathrm{Ru}(\mathrm{II})$ and Os(II) complexes Ru-3c and Os-3c (c standing for closed) and the corresponding open forms 3o, Ru-3o and Os-3o (see Chart 1). ${ }^{16}$ It was established for the closed-form molecules that the low-energy intraligand excited-state levels of the spiropyran moiety $\left(\mathrm{IL}_{\mathrm{Sp}}\right.$ ) lie above the lowest metalto-ligand charge transfer (MLCT) states of the chelated tris(bpy)-metal chromophores. On the other hand, the lowest triplet excited-state of the open-form spiropyran moiety $\left({ }^{3} \mathrm{IL}_{\mathrm{Mc}}\right)$ is situated between the lowest ${ }^{3}$ MLCT levels of the ruthenium and osmium polypyridyl moieties. Therefore, we might expect that for a Ru-BL-Os donor-bridge-acceptor system incorporating a closed spiropyran (Sp) moiety in the bridging ligand BL, energy transfer only takes place by a relatively inefficient superexchange mechanism (see Figure 1). When converted to the open merocyanine (Mc) form (see Scheme 1), however, the bridge will facilitate an efficient energy transfer by a hopping mechanism. This makes spiropyrans good candidates for the creation of a true energy transfer switch, in combination with $\left[\mathrm{Ru}(\mathrm{bpy})_{3}\right]^{2+}$-type donor and $\left[\mathrm{Os}(\mathrm{bpy})_{3}\right]^{2+}$-type acceptor termini.

To prove this concept, we studied a series of triad complexes containing a spiropyran-based bridge (BL), namely, two novel homodinuclear Ru-BL-Ru, one homodinuclear Os-BL-Os, and one heterodinuclear Ru-BL-Os, as depicted in Chart 1. Their syntheses, electrochemical and

(11) Guglielmetti, R. Photochromism: Molecules and Systems; 2nd ed.; Dürr, H., Bouas-Laurent, H., Eds.; Elsevier: Amsterdam, 2003; pp 314-466, and references therein.

(12) Bertelson, R. C. Organic Photochromic and Thermochromic Compounds; Crano, J. C., Guglielmetti, R. J., Eds.; Plenum: New York, 1999; Vol. 1, pp 11-83, and references therein.

(13) Berkovic, G.; Krongauz, V.; Weiss, V. Chem. Rev. 2000, 100, 17411753; and references therein.

(14) Appriou, P.; Guglielmetti, R.; Garnier, F. J. Photochem. 1978, 8, 145165 .

(15) Minkin, V. I. Chem. Rev. 2004, 104, 2751-2776.

(16) Jukes, R. T. F.; Bozic, B.; Hartl, F.; Belser, P.; De Cola, L. Inorg. Chem. 2006, 45, 8326-8341. photophysical properties are reported in this paper and compared with those of the related mononuclear $\mathrm{Ru}(\mathrm{II})$ and Os(II) bpy-nitrospiropyran complexes (see Chart 1) where appropriate.

\section{Experimental Section}

All chemicals and reagent grade products were purchased from Fluka, Aldrich, or Acros Chemicals and used without further purification, unless noted otherwise. The palladium catalyst $\left[\mathrm{Pd}(\mathrm{dppf})_{2} \mathrm{Cl}_{2}\right]\left(\mathrm{dppf}=1,1^{\prime}\right.$-bis(diphenylphosphino)ferrocene) (Strem Chemicals) was kept under argon. Column chromatography was performed using $230-400$ or $400-600$ mesh silica gel (Chemie Brunschwig AG). Purification by preparative plate chromatography was carried out on glass sheets recoated with $2 \mathrm{~mm}$ layers of silica gel $60 \mathrm{~F}_{254}$ purchased from Merck. Thin layer chromatography (TLC) was performed using aluminum sheets coated with silica gel $60 \mathrm{~F}_{254}$ (Merck).

We have previously described the synthesis of bpy-functionalized spiropyran ligand 4 (see Scheme 2), ${ }^{17,18}$ its derivative 5 (see Scheme $3)^{18}$ and complex $\mathbf{R u}\left(\boldsymbol{\mu}\right.$-2)Os (see Chart 1). ${ }^{18}$ Mononuclear complexes Ru-3c and Os-3c (see Chart 1) were also prepared following a protocol developed previously. ${ }^{16}$ The syntheses of the metal precursors $\left[\mathrm{Ru}(\mathrm{bpy})_{2} \mathrm{Cl}_{2}\right] \cdot \mathrm{H}_{2} \mathrm{O}$ and $\left[\mathrm{Os}(\mathrm{bpy})_{2} \mathrm{Cl}_{2}\right]$ followed an adapted general preparative route for compounds $[\mathrm{Ru}(\alpha-$ diimine $\left.)_{2} \mathrm{Cl}_{2}\right] .{ }^{19,20}$ All synthesized complexes were initially obtained as chloride salts and were isolated by precipitation with hexafluorophosphate anions. They were purified by preparative plate chromatography and obtained as racemic mixtures of $\Delta / \Lambda$ isomers. Elemental analyses were not carried out because of low reaction yields.

$5,6^{\prime}$-([2,2']-Bipyrid-4-yl)-1,3-dihydro-1,3,3-trimethylspiro[ $2 \mathrm{H}$ 1-benzopyran-2,2'-(2H)-indole] (1). (see Scheme 2). A solution of ligand $\mathbf{4}(150 \mathrm{mg} ; 0.27 \mathrm{mmol}), 2,2^{\prime}$-bipyridine-4-boronic acid (81 mg; $0.40 \mathrm{mmol}), \mathrm{Na}_{2} \mathrm{CO}_{3} \cdot 10 \mathrm{H}_{2} \mathrm{O}(42 \mathrm{mg} ; 0.40 \mathrm{mmol}$ ), and $\left[\mathrm{Pd}(\mathrm{dppf}) \mathrm{Cl}_{2}\right] \cdot \mathrm{CH}_{2} \mathrm{Cl}_{2}(9 \mathrm{mg} ; 5 \mathrm{~mol} \%)$ in dimethyl ether/water (3: $1 ; 4 \mathrm{~mL}$ ) was refluxed for $17 \mathrm{~h}$ under an argon atmosphere. It was then cooled to room temperature and poured into water $(50 \mathrm{~mL})$. The aqueous mixture was extracted with dichloromethane $(3 \times 50$ $\mathrm{mL}$ ). The combined extracts were dried over $\mathrm{MgSO}_{4}$ and concentrated under reduced pressure. The crude product was purified by flash chromatography $\left(\mathrm{SiO}_{2}, \mathrm{CH}_{2} \mathrm{Cl}_{2} / \mathrm{MeOH}(99: 1)\right)$. Yield $65 \mathrm{mg}$, $31 \% .{ }^{1} \mathrm{H}$ NMR $\left(500 \mathrm{MHz}, \mathrm{CDCl}_{3}\right): \delta 1.21(\mathrm{~s}, 3 \mathrm{H}, \mathrm{H}-\mathrm{C}(10)), 1.45$ (s, $3 \mathrm{H}, \mathrm{H}-\mathrm{C}(9)), 2.90(\mathrm{~s}, 3 \mathrm{H}, \mathrm{H}-\mathrm{C}(8)), 5.98\left(\mathrm{~d}, 1 \mathrm{H},{ }^{3} \mathrm{~J}=10.2\right.$, $\left.\mathrm{H}-\mathrm{C}\left(3^{\prime}\right)\right), 6.84\left(\mathrm{~d}, 1 \mathrm{H},{ }^{3} J=8.3, \mathrm{H}-\mathrm{C}(7)\right), 6.91\left(\mathrm{~d}, 1 \mathrm{H},{ }^{3} J=8.51\right.$, $\left.\mathrm{H}-\mathrm{C}\left(8^{\prime}\right)\right), 7.21\left(\mathrm{~d}, 1 \mathrm{H},{ }^{3} J=10.1, \mathrm{H}-\mathrm{C}\left(4^{\prime}\right)\right), 7.69-7.72(\mathrm{~m}, 2 \mathrm{H}$, $\mathrm{H}-\mathrm{C}\left(8^{\prime \prime}\right)$ and $\left.\mathrm{H}-\mathrm{C}\left(8^{\prime \prime \prime}\right)\right), 7.87\left(\mathrm{dd}, 1 \mathrm{H},{ }^{3} J=8.6,{ }^{4} J=2.5, \mathrm{H}-\mathrm{C}\left(7^{\prime}\right)\right)$, $7.93\left(\mathrm{dd}, 2 \mathrm{H},{ }^{3} \mathrm{~J}=5.5,{ }^{4} \mathrm{~J}=2.0, \mathrm{H}-\mathrm{C}\left(5^{\prime}\right)\right.$ and $\left.\mathrm{H}-\mathrm{C}(4)\right), 8.04(\mathrm{dd}$, $\left.1 \mathrm{H},{ }^{3} J=8.3,{ }^{4} J=2.0, \mathrm{H}-\mathrm{C}(6)\right), 8.12-8.25\left(\mathrm{~m}, 4 \mathrm{H}, \mathrm{H}-\mathrm{C}\left(2^{\prime \prime}\right)\right.$, $\left.\mathrm{H}-\mathrm{C}\left(2^{\prime \prime \prime}\right), \mathrm{H}-\mathrm{C}\left(9^{\prime \prime}\right), \mathrm{H}-\mathrm{C}\left(9^{\prime \prime \prime}\right)\right), 8.58\left(\mathrm{~d}, 1 \mathrm{H},{ }^{3} \mathrm{~J}=8.1, \mathrm{H}-\mathrm{C}\left(9^{\prime \prime}\right)\right)$, $8.62\left(\mathrm{~d}, 1 \mathrm{H},{ }^{3} J=8.1, \mathrm{H}-\mathrm{C}\left(9^{\prime \prime \prime}\right)\right), 8.65\left(\mathrm{~d}, 1 \mathrm{H},{ }^{3} J=6.3, \mathrm{H}-\mathrm{C}\left(4^{\prime \prime}\right)\right)$, 8.83-8.89 (m, 4H, H-C(7"), H-C(7'"), $\left.\mathrm{H}-\mathrm{C}\left(1^{\prime \prime}\right), \mathrm{H}-\mathrm{C}\left(1^{\prime \prime \prime}\right)\right)$. ${ }^{13} \mathrm{C}$ NMR (125 MHz, $\left.\mathrm{CDCl}_{3}\right): \delta 20.2,26.3,26.9,29.0,108.7,117.1$, $119.9,120.8,120.9,121.2,122.8,123.7,123.8,123.9,127.7,127.9$, 130.6, 130.7, 130.8, 130.9, 139.9, 140.2, 140.9, 150.0, 150.8. MS (ESI), $m / z: 620.22[\mathrm{M}+\mathrm{K}]^{+}$.

(17) Querol, M.; Bozic, B.; Salluce, N.; Belser, P. Polyhedron 2003, 22, 655-664.

(18) Belser, P.; De Cola, L.; Hartl, F.; Adamo, V.; Bozic, B.; Chriqui, Y.; Iyer, V. M.; Jukes, R. T. F.; Kuhni, J.; Querol, M.; Roma, S.; Salluce, N. Adv. Funct. Mater. 2006, 16, 195-208.

(19) Dwyer, F. P.; Hogarth, J. W. Inorganic Syntheses; McGraw-Hill: New York, 1957; Vol. 5, pp 206-207.

(20) Lay, P. A.; Sargeson, A. M.; Taube, H. Inorganic Syntheses; Shreeve, J. M.; Wiley: New York, 1986; Vol. 24, pp 291-299. 

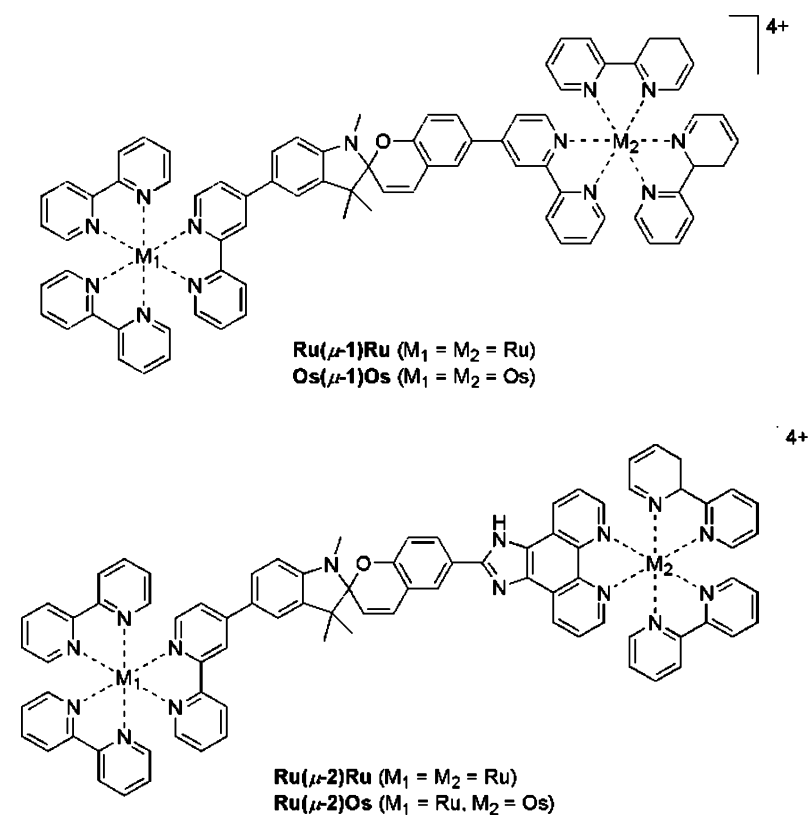

$\left[\mathbf{R u}(\mathbf{b p y})_{\mathbf{2}}(\boldsymbol{\mu}-\mathbf{1}) \mathbf{R u}(\mathbf{b p y})_{2}\right]\left(\mathbf{P F}_{\mathbf{6}}\right)_{\mathbf{4}}(\mathbf{R u}(\boldsymbol{\mu}-\mathbf{1}) \mathbf{R u})$. (see Scheme 2). A mixture of $\left[\mathrm{Ru}(\mathrm{bpy})_{2} \mathrm{Cl}_{2}\right] \cdot \mathrm{H}_{2} \mathrm{O}(42 \mathrm{mg} ; 0.085 \mathrm{mmol})$ and ligand $1(20 \mathrm{mg} ; 0.034 \mathrm{mmol})$ in ethanol/water $(3: 1 ; 6 \mathrm{~mL})$ was refluxed for $6 \mathrm{~h}$ under an argon atmosphere. After cooling, the solvent was distilled off under reduced pressure, and the residue was treated with $10 \%$ aq. $\mathrm{NH}_{4} \mathrm{PF}_{6}$. The precipitate was collected by filtration and washed with water. Purification of the crude product by plate chromatography on $\mathrm{SiO}_{2}$, using $\mathrm{MeCN} / \mathrm{MeOH} / \mathrm{KNO}_{3}$-solution (40: 10:10; $1 \mathrm{~g}$ of $\mathrm{KNO}_{3}$ in $10 \mathrm{~mL} \mathrm{H}_{2} \mathrm{O}$ ) as eluent gave an orange solid. Yield $10 \mathrm{mg}, 21 \%$. ${ }^{1} \mathrm{H}$ NMR (500 MHz, $\mathrm{CD}_{3} \mathrm{CN}$ ): $\delta 1.23$ (s, 3H, $\mathrm{H}-\mathrm{C}(10)), 1.37$ (s, 3H, H-C(9)), 2.82 (s, 3H, H-C(8)), 5.90 (d, $\left.1 \mathrm{H},{ }^{3} J=10.2, \mathrm{H}-\mathrm{C}\left(3^{\prime}\right)\right), 6.74\left(\mathrm{~d}, 1 \mathrm{H},{ }^{3} J=8.3, \mathrm{H}-\mathrm{C}(7)\right), 6.79(\mathrm{~d}$, $\left.1 \mathrm{H},{ }^{3} J=14.5, \mathrm{H}-\mathrm{C}\left(8^{\prime}\right)\right), 7.10\left(\mathrm{~d}, 1 \mathrm{H},{ }^{3} J=6.1, \mathrm{H}-\mathrm{C}\left(4^{\prime}\right)\right)$, 7.37-7.42 (m, 10H, H-C $\left.\left(2^{\mathrm{IV}}-2^{\mathrm{VII}}\right), \mathrm{H}-\mathrm{C}\left(8^{\prime \prime}-8^{\mathrm{VII}}\right)\right), 7.57-7.62(\mathrm{~m}$, $\left.3 \mathrm{H}, \mathrm{H}-\mathrm{C}\left(2^{\prime \prime}\right), \mathrm{H}-\mathrm{C}\left(2^{\prime \prime \prime}\right), \mathrm{H}-\mathrm{C}\left(5^{\prime}\right)\right), 7.66-7.68$ (m, 2H, H-C(4), $\left.\mathrm{H}-\mathrm{C}\left(7^{\prime}\right)\right), 7.72-7.82\left(\mathrm{~m}, 13 \mathrm{H}, \mathrm{H}-\mathrm{C}\left(3^{\mathrm{IV}}-3^{\mathrm{VII}}\right), \mathrm{H}-\mathrm{C}\left(4^{\prime \prime}\right), \mathrm{H}-\mathrm{C}\left(4^{\prime \prime \prime}\right)\right.$, $\left.\mathrm{H}-\mathrm{C}(6), \mathrm{H}-\mathrm{C}\left(9^{\prime \prime}-9^{\mathrm{VII}}\right)\right), 8.03-8.08\left(\mathrm{~m}, 10 \mathrm{H}, \mathrm{H}-\mathrm{C}\left(4^{\mathrm{IV}}-4^{\mathrm{VII}}\right) \mathrm{H}-\mathrm{C}\left(10^{\prime \prime}-\right.\right.$ $\left.\left.10^{\mathrm{VII}}\right)\right), 8.48-8.51\left(\mathrm{~m}, 8 \mathrm{H}, \mathrm{H}-\mathrm{C}\left(1^{\mathrm{IV}}-1^{\mathrm{VII}}\right), \mathrm{H}-\mathrm{C}\left(7^{\mathrm{IV}}-7^{\mathrm{VII}}\right)\right), 8.64-8.70$ $\left(\mathrm{m}, 4 \mathrm{H}, \mathrm{H}-\mathrm{C}\left(1^{\prime \prime}\right), \mathrm{H}-\mathrm{C}\left(1^{\prime \prime \prime}\right), \mathrm{H}-\mathrm{C}\left(7^{\prime \prime}\right), \mathrm{H}-\mathrm{C}\left(7^{\prime \prime \prime}\right)\right) .{ }^{13} \mathrm{C} \mathrm{NMR}(125$ $\left.\mathrm{MHz}, \mathrm{CD}_{3} \mathrm{CN}\right): \delta 20.0,26.0,29.0,52.7,106.2,108.3,116.5,120.6$, $120.8,120.9,120.9,121.5,121.7,121.9,124.4,124.8,125.2,125.2$, 127.0, 127.1, 128.4, 128.5, 128.5, 128.7, 128.9, 129.7, 129.9, 130.1,

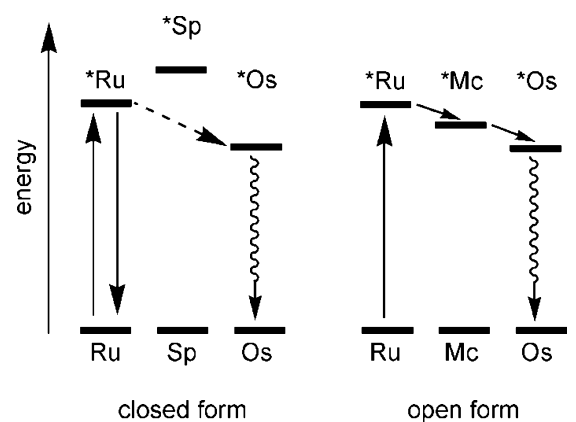

Figure 1. Energy diagram for the envisaged Ru-spiropyran-Os (donorbridge-acceptor) energy transfer switch. Left: Energy transfer by a superexchange mechanism occurs between the donor $(* \mathrm{Ru})$ and acceptor $(* \mathrm{Os})$ termini. The high-lying excited-state levels of the spiropyran $(* \mathrm{Sp})$ in the bridge do not facilitate the process. Right: Efficient energy transfer occurs between $* \mathrm{Ru}$ and $*$ Os through the intervening excited-state levels of the merocyanine $(* \mathrm{Mc})$.
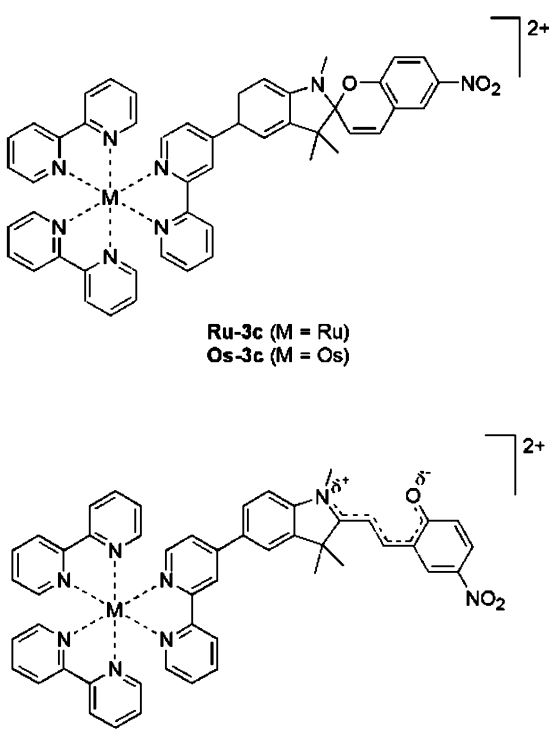

Ru-30 (M $=R u)$
Os-3o $(M=O s)$

$138.5,138.6,138.7,139.0,149.6,150.6,151.3,152.0,152.3,152.5$, 152.6, 157.0, 157.8, 157.9, 157.9, 157.9, 157.9, 158.1, 158.1, 158.2. MS (ESI), $m / z: 851.64\left[\left(\mathrm{M}-2 \mathrm{PF}_{6}\right) / 2\right]^{2+}, 519.44\left[\left(\mathrm{M}-3 \mathrm{PF}_{6}\right) /\right.$ $3]^{3+}, 353.34\left[\left(\mathrm{M}-4 \mathrm{PF}_{6}\right) / 4\right]^{4+}$.

[Os(bpy $)_{2}\left(\boldsymbol{\mu}\right.$-1)Os $\left.(\mathbf{b p y})_{2}\right]\left(\mathrm{PF}_{6}\right)_{4}(\mathbf{O s}(\boldsymbol{\mu}$-1)Os). (see Scheme 2). This dark green complex was prepared from $\left[\mathrm{Os}(\mathrm{bpy})_{2} \mathrm{Cl}_{2}\right](37 \mathrm{mg}$; $0.061 \mathrm{mmol})$ and ligand 1 (15 mg; $0.025 \mathrm{mmol})$ and purified by the same procedure as described above for $\left[\mathrm{Ru}(\mathrm{bpy})_{2}(\mu\right.$ 1) $\left.\mathrm{Ru}(\mathrm{bpy})_{2}\right]\left(\mathrm{PF}_{6}\right)_{4}$. Yield $15 \mathrm{mg}, 15 \%$. ${ }^{1} \mathrm{H}$ NMR $(500 \mathrm{MHz}$, $\mathrm{CD}_{3} \mathrm{CN}$ ): $\delta 1.22(\mathrm{~s}, 3 \mathrm{H}, \mathrm{H}-\mathrm{C}(10)), 1.36(\mathrm{~s}, 3 \mathrm{H}, \mathrm{H}-\mathrm{C}(9)), 2.82$ (s, $3 \mathrm{H}, \mathrm{H}-\mathrm{C}(8)), 5.90\left(\mathrm{~d}, 1 \mathrm{H},{ }^{3} \mathrm{~J}=10.3, \mathrm{H}-\mathrm{C}\left(3^{\prime}\right)\right), 6.73\left(\mathrm{~d}, 1 \mathrm{H},{ }^{3} \mathrm{~J}=\right.$ $8.3, \mathrm{H}-\mathrm{C}(7)), 6.79\left(\mathrm{~d}, 1 \mathrm{H},{ }^{3} J=8.4, \mathrm{H}-\mathrm{C}\left(8^{\prime}\right)\right), 7.09\left(\mathrm{~d}, 1 \mathrm{H},{ }^{3} \mathrm{~J}=\right.$ $\left.10.2, \mathrm{H}-\mathrm{C}\left(4^{\prime}\right)\right), 7.28-7.33\left(\mathrm{~m}, 10 \mathrm{H}, \mathrm{H}-\mathrm{C}\left(2^{\mathrm{IV}}-2^{\mathrm{VII}}\right), \mathrm{H}-\mathrm{C}\left(8^{\prime \prime}-8^{\mathrm{VII}}\right)\right)$, $7.48-7.57$ (m, 4H, H-C(2" $\left.\left.-2^{\prime \prime \prime}\right), \mathrm{H}-\mathrm{C}\left(5^{\prime}\right), \mathrm{H}-\mathrm{C}(4)\right), 7.63-7.75$ $\left(\mathrm{m}, \quad 14 \mathrm{H}, \mathrm{H}-\mathrm{C}\left(3^{\mathrm{IV}}-3^{\mathrm{VII}}\right), \mathrm{H}-\mathrm{C}\left(7^{\prime}\right), \mathrm{H}-\mathrm{C}(6), \mathrm{H}-\mathrm{C}\left(9^{\prime \prime}-9^{\mathrm{VII}}\right)\right)$, $7.83-7.90\left(\mathrm{~m}, 10 \mathrm{H}, \mathrm{H}-\mathrm{C}\left(4^{\mathrm{IV}}-4^{\mathrm{VII}}\right) \mathrm{H}-\mathrm{C}\left(10^{\prime \prime}-10^{\mathrm{VII}}\right)\right), 8.46-8.48$ $\left(\mathrm{m}, 8 \mathrm{H}, \mathrm{H}-\mathrm{C}\left(1^{\mathrm{IV}}-1^{\mathrm{VII}}\right), \mathrm{H}-\mathrm{C}\left(7^{\mathrm{IV}}-7^{\mathrm{VII}}\right)\right), 8.61-8.67\left(\mathrm{~m}, 4 \mathrm{H}, \mathrm{H}-\mathrm{C}\left(1^{\prime \prime}\right)\right.$, $\left.\mathrm{H}-\mathrm{C}\left(1^{\prime \prime \prime}\right), \mathrm{H}-\mathrm{C}\left(7^{\prime \prime}\right), \mathrm{H}-\mathrm{C}\left(7^{\prime \prime \prime}\right)\right) .{ }^{13} \mathrm{C}$ NMR $\left(125 \mathrm{MHz}, \mathrm{CD}_{3} \mathrm{CN}\right)$ : $\delta$ 20.0, 26.1, 29.0, 52.7, 108.2, 116.4, 120.5, 120.9, 121.6, 121.8, $121.9,124.7,125.2,125.4,125.4,126.8,127.2,128.4,128.9,129.0$, $129.9,130.1,137.9,137.9,137.9,138.0,138.9,148.9,150.0,151.2$, 151.4, 151.5, 151.6, 151.6, 151.7, 157.1, 159.9, 159.9, 159.9, 160.1. MS (ESI), $m / z: 940.69\left[\left(\mathrm{M}-2 \mathrm{PF}_{6}\right) / 2\right]^{2+}, 578.80\left[\left(\mathrm{M}-3 \mathrm{PF}_{6}\right) /\right.$ $3]^{3+}, 397.86\left[\left(\mathrm{M}-4 \mathrm{PF}_{6}\right) / 4\right]^{4+}$.

5-(2,2'-Bipyrid-4-yl)-1,3-dihydro-6' $-([1,10]$ phenanthroline[5,6d]imidazol-2-yl)-1,3,3-trimethylspiro[2H-1-benzopyran-2,2'-(2H)indole] (2). (see Scheme 3). A mixture of ligand 5 (100 mg; 0.218 $\mathrm{mmol}$ ), 1,10-phenanthroline-5,6-dione (54 mg; $0.262 \mathrm{mmol}$ ), and ammonium acetate $(261 \mathrm{mg})$ in ethanol $(80 \mathrm{~mL})$ was refluxed for $20 \mathrm{~h}$ under an argon atmosphere. The reaction was monitored by TLC $\left(\mathrm{SiO}_{2} ; \mathrm{CH}_{2} \mathrm{Cl}_{2} / \mathrm{MeOH} / \mathrm{Et}_{3} \mathrm{~N}\right.$ (96:4:0.25)). After cooling, the solvent was distilled off, and the residue was treated with water (2 $\mathrm{mL}$ ). The precipitate was collected by filtration, washed with water and vacuum-dried. Purification of the crude mixture by column chromatography $\left(\mathrm{SiO}_{2} ; \mathrm{CH}_{2} \mathrm{Cl}_{2} / \mathrm{MeOH}(99: 2)\right)$ gave a blue solid. Yield $90 \mathrm{mg}, 64 \%$. ${ }^{1} \mathrm{H} \mathrm{NMR}\left(500 \mathrm{MHz}, \mathrm{CDCl}_{3}\right): \delta 1.27(\mathrm{~s}, 3 \mathrm{H}$, $\mathrm{H}-\mathrm{C}(10)), 1.44$ (s, 3H, H-C(9)), 2.85 (s, 3H, H-C(8)), 5.84 (d, $\left.1 \mathrm{H},{ }^{3} J=10.2, \mathrm{H}-\mathrm{C}\left(3^{\prime}\right)\right), 6.64\left(\mathrm{~d}, 1 \mathrm{H},{ }^{3} J=8.1, \mathrm{H}-\mathrm{C}(7)\right), 6.90(\mathrm{~d}$, $\left.1 \mathrm{H},{ }^{3} J=8.51, \mathrm{H}-\mathrm{C}\left(8^{\prime}\right)\right), 7.04\left(\mathrm{~d}, 1 \mathrm{H},{ }^{3} J=10.1, \mathrm{H}-\mathrm{C}\left(4^{\prime}\right)\right), 7.30$ 
Scheme 2. Synthesis of Spiropyran Ligand $\mathbf{1}$ and Corresponding Dinuclear Complexes $\mathbf{R u}(\boldsymbol{\mu}-\mathbf{1}) \mathbf{R u}\left(\mathrm{M}_{1}=\mathrm{M}_{2}=\mathrm{Ru}\right)$ and $\mathbf{O s}(\boldsymbol{\mu}-\mathbf{1}) \mathbf{O s}\left(\mathrm{M}_{1}=\mathrm{M}_{2}=\mathrm{Os}\right)$
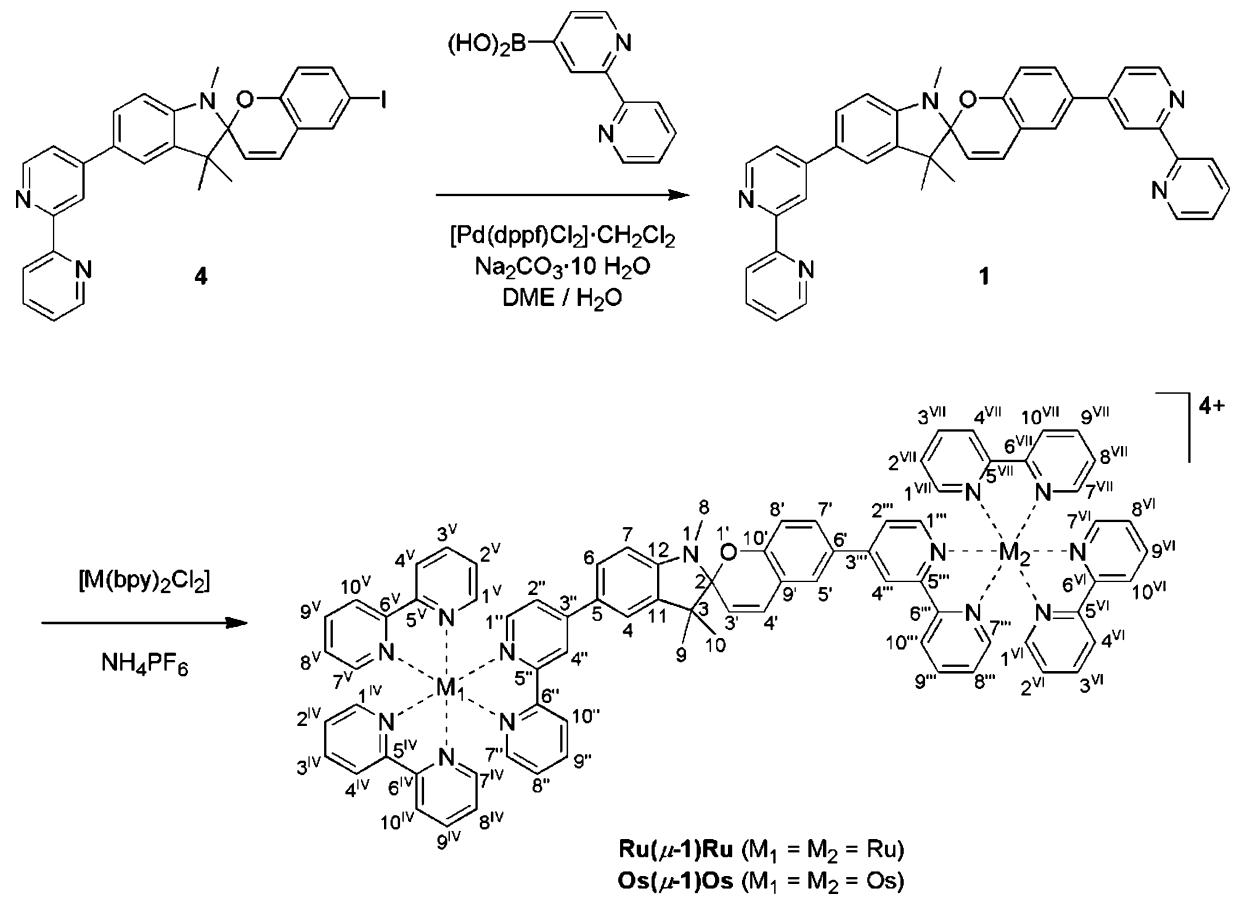

Scheme 3. Synthesis of Spiropyran Ligand 2 and Corresponding Diruthenium Complex $\mathbf{R u}(\boldsymbol{\mu}$-2)Ru
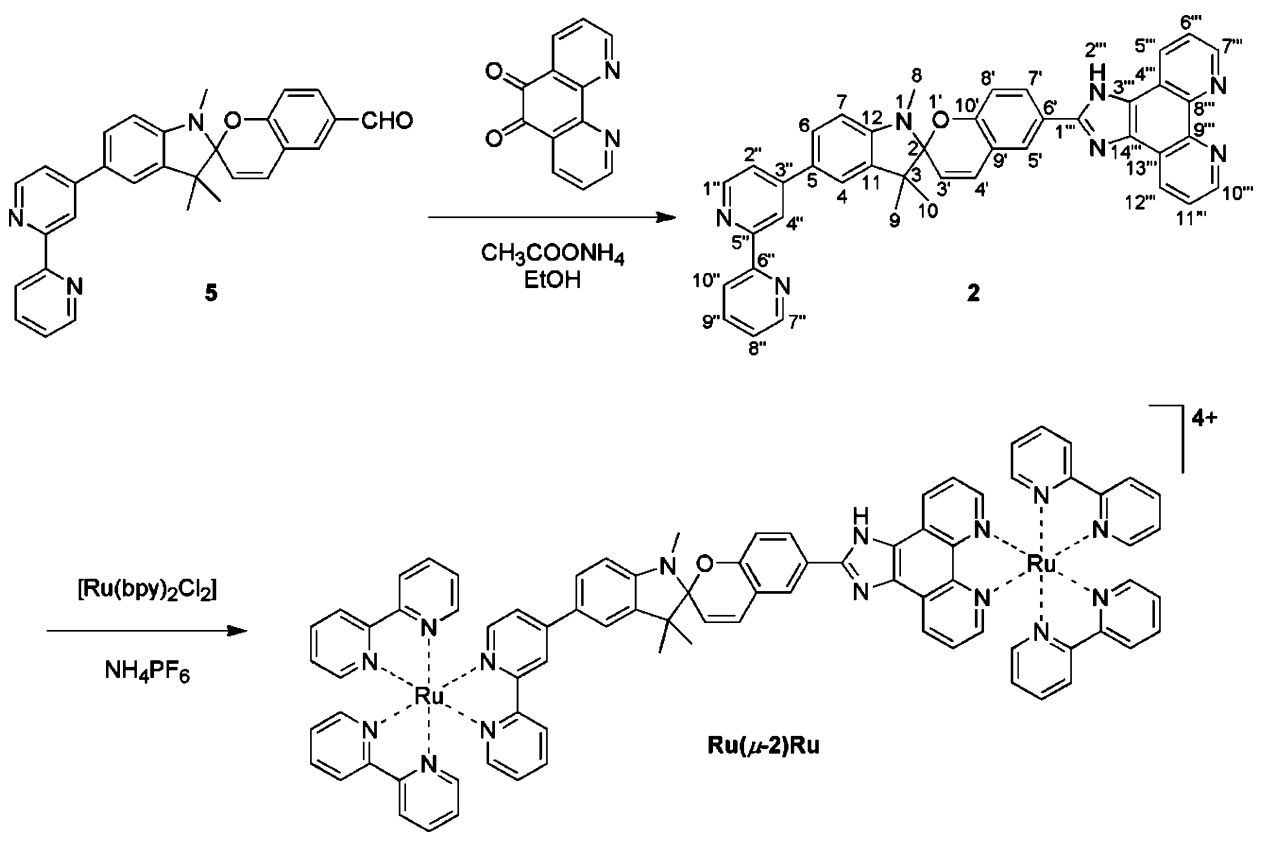

(ddd, $\left.1 \mathrm{H},{ }^{3} \mathrm{~J}=7.5,{ }^{3} \mathrm{~J}=4.6,{ }^{4} \mathrm{~J}=1.3, \mathrm{H}-\mathrm{C}\left(8^{\prime \prime}\right)\right), 7.54-7.55(\mathrm{~m}$, $\left.2 \mathrm{H}, \mathrm{H}-\mathrm{C}\left(2^{\prime \prime}\right), \mathrm{H}-\mathrm{C}(4)\right), 7.65-7.71\left(\mathrm{~m}, 3 \mathrm{H}, \mathrm{H}-\mathrm{C}\left(6^{\prime \prime \prime}\right), \mathrm{H}-\mathrm{C}(6)\right.$ and $\left.\mathrm{H}-\mathrm{C}\left(11^{\prime \prime \prime}\right)\right), 7.81-7.84\left(\mathrm{~m}, 1 \mathrm{H}, \mathrm{H}-\mathrm{C}\left(9^{\prime \prime}\right)\right), 8.04-8.08(\mathrm{~m}$, $2 \mathrm{H}, \mathrm{H}-\mathrm{C}\left(5^{\prime}\right)$ and $\left.\mathrm{H}-\mathrm{C}\left(7^{\prime}\right)\right), 8.42\left(\mathrm{dt}, 1 \mathrm{H},{ }^{3} \mathrm{~J}=8.1,{ }^{4} \mathrm{~J}=1.1\right.$, $\left.\mathrm{H}-\mathrm{C}\left(10^{\prime \prime}\right)\right), 8.53\left(\mathrm{dd}, 1 \mathrm{H},{ }^{3} J=8.1,{ }^{4} J=1.7, \mathrm{H}-\mathrm{C}\left(5^{\prime \prime \prime}\right)\right.$ or $\left.\mathrm{H}-\mathrm{C}\left(12^{\prime \prime \prime}\right)\right), 8.63\left(\mathrm{dd}, 1 \mathrm{H},{ }^{4} J=1.8,{ }^{5} J=0.7, \mathrm{H}-\mathrm{C}\left(4^{\prime \prime}\right)\right), 8.66$ $\left(\mathrm{dd}, 1 \mathrm{H},{ }^{3} \mathrm{~J}=5.2,{ }^{5} \mathrm{~J}=0.7, \mathrm{H}-\mathrm{C}\left(1^{\prime \prime}\right)\right), 8.71\left(\mathrm{ddd}, 1 \mathrm{H},{ }^{3} \mathrm{~J}=4.6,{ }^{4} \mathrm{~J}\right.$ $\left.=1.8,{ }^{5} \mathrm{~J}=0.9, \mathrm{H}-\mathrm{C}\left(7^{\prime \prime}\right)\right), 8.82\left(\mathrm{dd}, 1 \mathrm{H},{ }^{3} \mathrm{~J}=8.1,{ }^{4} \mathrm{~J}=1.8\right.$, $\mathrm{H}-\mathrm{C}\left(5^{\prime \prime \prime}\right)$ or $\left.\mathrm{H}-\mathrm{C}\left(12^{\prime \prime \prime}\right)\right), 9.15$ (m, 2H, $\mathrm{H}-\mathrm{C}\left(7^{\prime \prime}\right)$ and $\left.\mathrm{H}-\mathrm{C}\left(10^{\prime \prime \prime}\right)\right)$. ${ }^{13} \mathrm{C}$ NMR $\left(125 \mathrm{MHz}, \mathrm{CDCl}_{3}\right): \delta 20.1,20.9,25.9,28.9,52.0,105.3$, 107.1, 115.8, 117.8, 118.2, 119.1, 119.2, 120.1, 120.4, 120.8, 121.4, 122.7, 123.1, 123.3, 123.7, 125.9, 127.2, 128.3, 129.1, 129.1, 129.2, 130.6, 134.7, 136.9, 137.4, 142.8, 144.3, 144.6, 148.9, 149.1, 149.2,
Chart 2
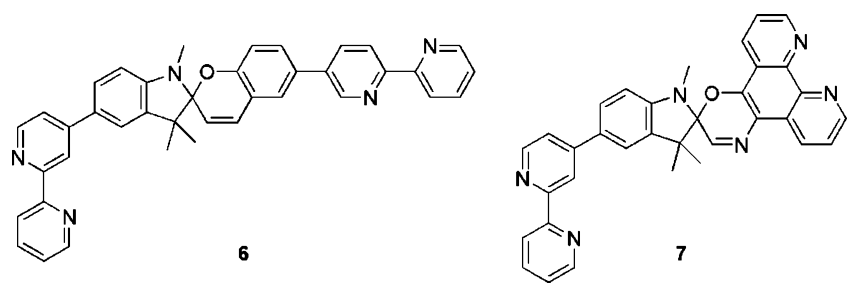

149.2, 149.3, 149.7, 156.1, 157.1, 163.5. MS (ESI), $\mathrm{m} / \mathrm{z}: 651.25$ $[\mathrm{M}+\mathrm{H}]^{+}$.

$\left[\mathbf{R u}(\text { bpy })_{2}(\boldsymbol{\mu} \text {-2)Ru(bpy })_{2}\right]\left(\mathbf{P F}_{\mathbf{6}}\right)_{\mathbf{4}}(\mathbf{R u}(\boldsymbol{\mu}$-2) Ru). (see Scheme 3). $\left[\mathrm{Ru}(\mathrm{bpy}){ }_{2} \mathrm{Cl}_{2}\right] \cdot \mathrm{H}_{2} \mathrm{O}(50 \mathrm{mg} ; 0.769 \mathrm{mmol})$, ligand 2 (88 mg; 0.169 $\mathrm{mmol})$, and 2-methoxyethanol/ $\mathrm{H}_{2} \mathrm{O}(95: 5 ; 3 \mathrm{~mL})$ were refluxed for 


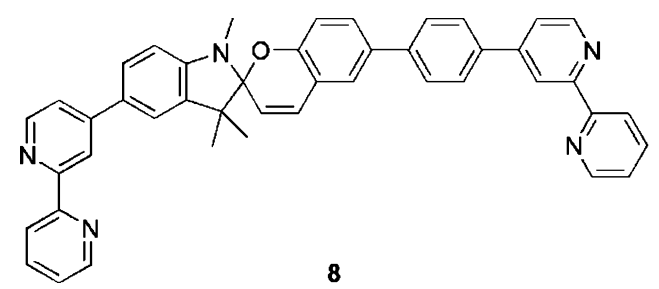

$5 \mathrm{~h}$ under argon atmosphere. The solvent was distilled off under reduced pressure, and the residue was treated with aqueous $10 \%$ $\mathrm{NH}_{4} \mathrm{PF}_{6}$. The precipitate was collected by filtration and washed with water. The crude product was purified by column chromatography (neutral $\mathrm{Al}_{2} \mathrm{O}_{3}$ ), using a mixture of acetone $/ \mathrm{H}_{2} \mathrm{O}(99: 1)$ as eluent. The final purification by plate chromatography on $\mathrm{SiO}_{2}$, using $\mathrm{MeCN} / \mathrm{MeOH} / \mathrm{KNO}_{3}$-solution (40:10:10; $1 \mathrm{~g}$ of $\mathrm{KNO}_{3}$ in $10 \mathrm{~mL}$ of $\mathrm{H}_{2} \mathrm{O}$ ) as eluent gave a red solid. Yield $12 \mathrm{mg}, 10 \%$. ${ }^{1} \mathrm{H}$ NMR $\left(500 \mathrm{MHz}, \mathrm{CD}_{3} \mathrm{CN}\right): \delta 1.26(\mathrm{~s}, 3 \mathrm{H}), 1.41(\mathrm{~s}, 3 \mathrm{H}), 2.86(\mathrm{~s}, 3 \mathrm{H})$, $5.97\left(\mathrm{~d}, 1 \mathrm{H},{ }^{3} \mathrm{~J}=10.3\right), 6.77\left(\mathrm{~d}, 1 \mathrm{H},{ }^{3} \mathrm{~J}=8.4\right), 6.89\left(\mathrm{~d}, 1 \mathrm{H},{ }^{3} \mathrm{~J}=\right.$ 8.6), 7.19-7.23 (m, 3H), 7.39-7.47 (m, 8H), 7.54-7.59 (m, 2H), $7.60-7.64(\mathrm{~m}, 2 \mathrm{H}), 7.69\left(\mathrm{t}, 1 \mathrm{H},{ }^{4} \mathrm{~J}=1.6\right), 7.74-7.84(\mathrm{~m}, 10 \mathrm{H})$, $7.97-8.01(\mathrm{~m}, 2 \mathrm{H}), 8.04-8.13(\mathrm{~m}, 9 \mathrm{H}), 8.18\left(\mathrm{dd}, 1 \mathrm{H},{ }^{3} \mathrm{~J}=8.6,{ }^{4} \mathrm{~J}\right.$ $=1.8), 8.25\left(\mathrm{~d}, 1 \mathrm{H},{ }^{4} J=2.2\right), 8.49-8.55(\mathrm{~m}, 8 \mathrm{H}), 8.70(\mathrm{bdd}, 1 \mathrm{H}$, $\left.{ }^{3} J=4.9,{ }^{4} J=3.3\right), 8.88\left(\mathrm{dd}, 1 \mathrm{H},{ }^{3} J=8.4,{ }^{4} J=1.3\right), 8.98(\mathrm{dd}$, $\left.1 \mathrm{H},{ }^{3} J=8.2,{ }^{4} J=1.3\right) .{ }^{13} \mathrm{C} \mathrm{NMR}\left(125 \mathrm{MHz}, \mathrm{CD}_{3} \mathrm{CN}\right): \delta 20.1$, 26.2, 29.2, 30.8, 30.9, 30.9, 52.9, 106.6, 108.5, 116.8, 119.7, 119.7, $119.8,120.7,121.1,121.2,121.6,121.8,124.5,125.2,125.3,125.3$, $125.9,127.2,127.5,127.5,127.6,128.4,128.5,128.5,128.5,128.6$, 128.6, 120.0, 130.1, 130.2, 130.5, 132.1, 137.6, 138.6, 138.7, 138.7, $138.8,138.9,138.9,130.1,144.9,146.9,147.6,150.7,151.4,152.1$, $152.6,152.6,152.8,152.9,152.9,152.9,153.0,157.9,158.0,158.1$, $158.2,158.3,158.5,166.5$. MS (ESI), $m / z: 884.14\left[\left(\mathrm{M}-2 \mathrm{PF}_{6}\right) /\right.$ $2]^{2+}, 541.10\left[\left(\mathrm{M}-3 \mathrm{PF}_{6}\right) / 3\right]^{3+}, 369.58\left[\left(\mathrm{M}-4 \mathrm{PF}_{6}\right) / 4\right]^{4+}$.

\section{General Techniques}

All products were characterized by ${ }^{1} \mathrm{H}$ NMR and ${ }^{13} \mathrm{C}$ NMR spectra obtained with a Bruker Advance DRX-500 (500 MHz for ${ }^{1} \mathrm{H}$ NMR and $125.75 \mathrm{MHz}$ for ${ }^{13} \mathrm{C}$ ) spectrometer. Chemical shifts are given in ppm, using the solvent itself as internal standard. The chemical shifts are expressed as $\delta$ values and the coupling constants $(J)$ are given in hertz. The numbers in the assignment of the NMR signals refer to the numbering of atoms presented in Schemes 2 and 3.

ESI mass spectra were recorded on a Bruker FTMS 4.7T BioAPEXII spectrometer, UV-vis absorption spectra on a Hewlett-Packard 8453 diode array spectrophotometer and steady-state emission spectra on a Spex 1680 spectrofluorimeter. Emission spectra were not corrected for the photomultiplier response. Deaerated solutions were prepared by the freeze-pump-thaw technique on a vacuum line. Luminescence quantum yields were determined for optically diluted solutions, using aerated solutions of $\left[\mathrm{Ru}(\mathrm{bpy})_{3}\right] \mathrm{Cl}_{2}$ in $\mathrm{H}_{2} \mathrm{O}\left(\varphi_{\mathrm{em}}=0.028\right)^{21}$ or $\left[\mathrm{Os}(\mathrm{bpy})_{3}\right]\left(\mathrm{PF}_{6}\right)_{2}$ in $\mathrm{MeCN}\left(\varphi_{\mathrm{em}}\right.$ $=0.0035)^{22}$ as references where appropriate.

Nanosecond time-resolved absorption spectra were measured using a setup described previously. ${ }^{23}$ The irradiation

(21) Nakamaru, K. Bull. Chem. Soc. Jpn. 1982, 55, 2697-2705.

(22) Frank, M.; Nieger, M.; Vögtle, F.; Belser, P.; von Zelewsky, A.; De Cola, L.; Balzani, V.; Barigelletti, F.; Flamigni, L. Inorg. Chim. Acta 1996, 242, 281-291.

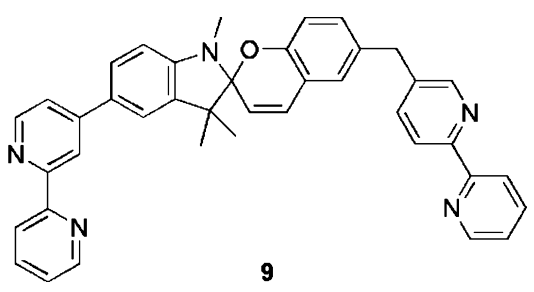

source was a continuously tunable $(400-700 \mathrm{~nm})$ Coherent Infinity XPO laser (2 ns fwhm). A 50\% mirror was used to divide the probe light into sample and reference beams, which greatly improved the signal-to-noise ratio.

Time-resolved emission studies in the nanosecond time domain were performed using a continuously tunable $(400-700 \mathrm{~nm})$ Coherent Infinity XPO laser (2 ns fwhm) as the excitation source. The emitted light was detected using a streak camera (Hamamatsu C5680-21) equipped with an M 5677 sweep unit over a range of $300 \mathrm{~nm}$, or at a single emission wavelength, using a combination of an Oriel monochromator and a Hamamatsu P28 photomultiplier tube, from which the signal was recorded on a Tektronix TDS684B $\left(1 \mathrm{GHz}, 5 \mathrm{GS} \mathrm{s}^{-1}\right)$ oscilloscope.

(Sub)picosecond transient absorption spectra were measured using a setup described in detail in a previous paper. ${ }^{24}$ The laser part is based on a Spectra-Physics Hurrican Tisapphire regenerative amplifier system, and the full spectrum setup on an optical parametric amplifier (Spectra-Physics OPA 800) as a pump. A residual fundamental light from the pump OPA is used for the generation of white light that is detected with a CCD spectrograph. The laser output is typically $5 \mu \mathrm{J}$ pulse $^{-1}$ (130 fs fwhm) with a repetition rate of $1 \mathrm{kHz}$. A circular cuvette $(d=1.8 \mathrm{~cm}, l=1 \mathrm{~mm}$, Hellma) containing the sample solution was placed in a homemade rotating ball bearing (1000 rpm) to avoid local heating by the laser beam and buildup of a photoproduct.

Irradiation experiments were performed using a highpressure Hg lamp (Oriel Model 6137) in combination with appropriate interference filters. The incidental photon flux was determined before and after each measurement, using fresh solutions of Aberchrome $540\left(\varphi_{\lambda}=0.20\right.$ for $313 \mathrm{~nm}$ $<\lambda<366 \mathrm{~nm})^{25}$ and Aberchrome 540P $\left(\varphi_{\lambda}=0.178-2.40\right.$ $\times 10^{-4} \cdot \lambda$ for $\left.440 \mathrm{~nm}<\lambda<550 \mathrm{~nm}\right)^{26}$ actinometers in toluene. The potential conversion of the spiropyrans was investigated by UV-vis spectroscopy, monitoring the visible spectral region for appearance of the characteristic absorption band of the merocyanine photoproducts.

Cyclic and Osteryoung square-wave voltammograms of approximately $10^{-4} \mathrm{M}$ parent compounds in $10^{-1} \mathrm{M} \mathrm{Bu}_{4} \mathrm{NPF}_{6}$

(23) Kleverlaan, C. J.; Stufkens, D. J.; Clark, I. P.; George, M. W.; Turner, J. J.; Martino, D. M.; van Willigen, H.; Vlček, A., Jr. J. Am. Chem. Soc. 1998, 120, 10871-10879.

(24) Campagna, S.; Serroni, S.; Puntoriero, F.; Loiseau, F.; De Cola, L.; Kleverlaan, C. J.; Becher, J.; Sorensen, A. P.; Hascoat, P.; Thorup, N. Chem. Eur. J. 2002, 8, 4461-4469.

(25) Heller, H. G.; Langan, J. R. J. Chem. Soc., Perkin Trans. 2 1981, 341-343.

(26) Glaze, A. P.; Heller, H. G.; Morgan, C. J.; Oliver, S. N.; Whittall, J. Proc. Xth IUPAC Symposium on Photochemistry; Interlaken, Switzerland, 1984, 369-370. See also: Kuhn, H. J.; Braslavsky, S. E.; Schmidt, R. Pure Appl. Chem. 2004, 76, 2105-2146. 
electrolyte solution were recorded in a gastight singlecompartment three-electrode cell equipped with platinum disk working (apparent surface of $0.42 \mathrm{~mm}^{2}$ ), coiled platinum wire auxiliary, and silver wire pseudoreference electrodes. The cell was connected to a computer-controlled PAR Model 283 potentiostat. All redox potentials are reported against the ferrocene/ferrocenium $\left(\mathrm{Fc} / \mathrm{Fc}^{+}\right)$redox couple used as internal standard. $^{27,28}$

UV-vis spectroelectrochemical experiments were performed in an airtight optically transparent thin-layer electrochemical (OTTLE) cell, ${ }^{29}$ equipped with a Pt minigrid working electrode (32 wires $\mathrm{cm}^{-1}$ ) and $\mathrm{CaF}_{2}$ or quartz windows. The controlled-potential electrolyses were carried out with a PA4 potentiostat (EKOM, Polná, Czech Republic).

Estimated experimental errors in the reported data are as follows: absorption and emission maxima, $\pm 2 \mathrm{~nm}$; emission lifetimes, $\pm 8 \%$; emission quantum yields, $\pm 20 \%$; ns TA lifetimes, $\pm 20 \%$; electrode potentials, $\pm 5 \mathrm{mV}$.

\section{Results and Discussion}

Synthesis. The synthesis of a dinuclear complex bridged by a photochromic spiropyran/spirooxazine-based bridging ligand proved to be a challenging exercise. Initially, spiropyran-based ligand 6 and spirooxazine-based ligand 7 were synthesized (see Chart 2), both carrying two chelating ligands for metal coordination. ${ }^{17}$ Unfortunately, these two ligands were unstable under the conditions of metal complex formation. ESI-MS analyses showed that decomposition occurred around the spiro-carbon atom. As Ru- and Os-metal fragments had earlier been complexed successfully to a $2,2^{\prime}$ bipyridine moiety attached to the indoline half of a nitrospiropyran, ${ }^{16}$ it was assumed that the decomposition was related to the complexation at the pyran/oxazine side.

In an attempt to prepare a stable ligand for bimetallic complexation, spiropyran $\mathbf{6}$ was modified by introducing phenylene and methylene spacer groups between the pyran half of the switching unit and the 2,2'-bipyridine moiety, resulting in ligands $\mathbf{8}$ and $\mathbf{9}$, respectively (see Chart 3 ). In both cases the stability of the uncomplexed ligands decreased drastically and isolation of pure compounds proved impossible.

We therefore decided to change the synthetic approach and introduce a less electron-donating type of substituent on the pyran part of the switching unit. This approach indeed led to improved inherent stability of the ligands and opened roads to metal complexation.

First ligand 1 was synthesized by a $\left[\mathrm{PdCl}_{2}(\mathrm{dppf})\right]$-catalyzed Suzuki cross-coupling reaction of spiropyran $\mathbf{4}$ with boronic acid (see Scheme 2). The preparation of precursor $\mathbf{4}$ followed a previously developed protocol. ${ }^{17,18}$ Mononitration of commercially available 2,2'-bipyridine- $N$-oxide with $\mathrm{NaNO}_{3}$ in concentrated $\mathrm{H}_{2} \mathrm{SO}_{4}$ yielded 4-nitro-2,2' -bipyridine- $N$-oxide that was further converted into 4-bromo-2,2'-bipyridine in

(27) Gritzner, G.; Kota, J. Pure Appl. Chem. 1984, 56, 461-466.

(28) Pavlishchuk, V. V.; Addison, A. W. Inorg. Chim. Acta 2000, 298, 97-102.

(29) Krejčík, M.; Daněk, M.; Hartl, F. J. Electroanal. Chem. 1991, 317, 179-187. the presence of $\mathrm{PBr}_{3}{ }^{30-32}$ The 2,2'-bipyridine-4-boronic acid was obtained using a classical reaction with $n \mathrm{BuLi}$ and subsequent treatment with trimethylboran in diethyl ether at low temperature. ${ }^{33}$

With ligand 1 in hand, for the first time, it proved possible to perform a double complexation reaction on a spiropyrantype ligand, which did not result in decomposition. Homodinuclear complexes $\mathbf{R u}(\boldsymbol{\mu}-\mathbf{1}) \mathbf{R u}$ and $\mathbf{O s}(\boldsymbol{\mu}$-1)Os (see Scheme 2) were synthesized by a complexation reaction of ligand $\mathbf{1}$ with the metal complex precursors $\left[\mathrm{Ru}(\mathrm{bpy})_{2} \mathrm{Cl}_{2}\right] \cdot 2 \mathrm{H}_{2} \mathrm{O}$ and $\left[\mathrm{Os}(\mathrm{bpy})_{2} \mathrm{Cl}_{2}\right]$, respectively.

The synthesis of the corresponding heterodinuclear $\mathrm{Ru} / \mathrm{Os}$ complex with bridging ligand $\mathbf{1}$ was not successful. Our synthetic strategy was based on the chemistry-on-thecomplex protocol, in which classical organic and organometallic reactions are performed directly on the metal complexes. Unfortunately the yield of the Suzuki crosscoupling reaction between precursors $\left[\mathrm{Ru}(\mathrm{bpy})_{2}(\mathbf{4})\right]^{2+}$ and $\left[\mathrm{Os}(\mathrm{bpy})_{2}\left(\mathrm{bpy}-\mathrm{B}(\mathrm{OH})_{2}\right)\right]^{2+}$ was very low.

To overcome these synthetic difficulties in preparing a heterodinuclear metal complex, we replaced the Suzuki crosscoupling step by a coupling reaction between an aromatic aldehyde and phenanthroline-5,6-dione (pd) (see Scheme 3). ${ }^{34}$ First, ligand 5 was reacted with $\left[\mathrm{Ru}(\text { bpy })_{2} \mathrm{Cl}_{2}\right] \cdot 2 \mathrm{H}_{2} \mathrm{O}$ to form $\left[\mathrm{Ru}(\mathrm{bpy})_{2}(\mathbf{5})\right]\left(\mathrm{PF}_{6}\right)_{2}$. This complex was coupled with the pd moiety, and the resulting complex $\left[\mathrm{Ru}(\mathrm{bpy})_{2}(\mathbf{2})\right]\left(\mathrm{PF}_{6}\right)_{2}$ was then reacted with $\left[\mathrm{Os}(\mathrm{bpy})_{2} \mathrm{Cl}_{2}\right]$ to yield $\mathbf{R u}(\boldsymbol{\mu}-\mathbf{2}) \mathbf{O s}$. The experimental details of this reaction have been published in the literature. ${ }^{17,18}$

The corresponding homodinuclear complex $\mathbf{R u}(\boldsymbol{\mu}-\mathbf{2}) \mathbf{R u}$ was synthesized by the complexation reaction of the new spiropyran ligand $\mathbf{2}$ with two molar equivalents of the complex precursor $\left[\mathrm{Ru}(\mathrm{bpy})_{2} \mathrm{Cl}_{2}\right] \cdot 2 \mathrm{H}_{2} \mathrm{O}$. This ligand was formed by a coupling reaction of 6-formyl-functionalized spiropyran 5 with 1,10-phenanthroline-5,6-dione (see Scheme 3 ). The preparation of ligand $\mathbf{5}$ followed a previously developed protocol. ${ }^{17,18}$ The reactant 1,10 -phenanthroline5,6-dione was prepared by oxidation of 1,10-phenanthroline in a mixture of concentrated $\mathrm{H}_{2} \mathrm{SO}_{4}$, concentrated $\mathrm{HNO}_{3}$ and $\mathrm{KBr}^{35,36}$

Electronic Absorption Spectra. Electronic absorption spectra of dinuclear complexes $\operatorname{Ru}(\boldsymbol{\mu}-\mathbf{1}) \mathbf{R u}, \mathbf{O s}(\boldsymbol{\mu}-\mathbf{1}) \mathbf{O s}$, $\operatorname{Ru}(\boldsymbol{\mu}-2) \mathbf{R u}$, and $\operatorname{Ru}(\boldsymbol{\mu}-\mathbf{2}) \mathbf{O s}$ in acetonitrile $(\mathrm{MeCN})$ are shown in Figure 2. Absorption maxima and the corresponding molar absorption coefficients $\left(\varepsilon_{\max }\right)$ are reported in Table 1.

The UV-vis absorption spectra of all investigated complexes exhibit essentially the same features. The bands centered around 450-460 $\mathrm{nm}$ can be assigned to metal-to-

(30) Wenkert, D.; Woodward, R. B. J. Org. Chem. 1983, 48, 283-289.

(31) Cook, M. J.; Lewis, A. P.; McAuliffe, G. S. G.; Skarda, V.; Thomson, A. J.; Glasper, J. L.; Robbins, D. J. J. Chem. Soc., Perkin Trans. 2 1984, 1293-1301.

(32) Aspley, C. J.; Williams, J. A. G. New J. Chem. 2001, 25, 1136-1147.

(33) Morris, G. A.; Nguyen, S. T. Tetrahedron Lett. 2001, 42, 2093-2096.

(34) Amouyal, E.; Homsi, A.; Chambron, J. C.; Sauvage, J. P. J. Chem. Soc., Dalton Trans. 1990, 1841-1845.

(35) Yamada, M.; Tanaka, Y.; Yoshimoto, Y.; Kuroda, S.; Shimao, I. Bull. Chem. Soc. Jpn. 1992, 65, 1006-1011.

(36) Gholamkhass, B.; Koike, K.; Negishi, N.; Hori, H.; Sano, T.; Takeuchi, K. Inorg. Chem. 2003, 42, 2919-2932. 


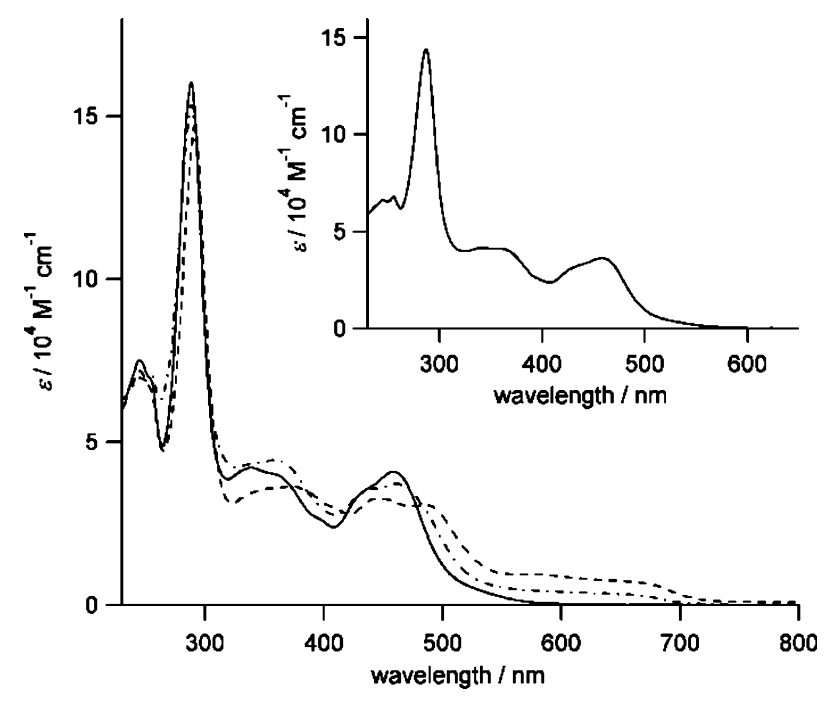

Figure 2. UV-vis absorption spectra of $\mathbf{R u}(\boldsymbol{\mu}-\mathbf{1}) \mathbf{R u}$ (solid line), $\mathbf{O s}(\boldsymbol{\mu}$ 1)Os (dashed line), $\mathbf{R u}(\mu-2) O s$ (dashed-dotted line), and $\mathbf{R u}(\boldsymbol{\mu}-\mathbf{2}) \mathbf{R u}$ (inset) recorded in $\mathrm{MeCN}$ at $293 \mathrm{~K}$.

Table 1. Absorption Maxima and Molar Absorption Coefficients of the Investigated Dinuclear Complexes $\mathbf{R u}(\boldsymbol{\mu}-\mathbf{1}) \mathbf{R u}, \mathbf{O s}(\boldsymbol{\mu}-\mathbf{1}) \mathbf{O} \mathbf{s}, \mathbf{R u}(\boldsymbol{\mu}-\mathbf{2}) \mathbf{R u}$, and $\mathbf{R u}(\mu-2) \mathbf{O s}^{a}$

complex $\quad \lambda_{\max } / \mathrm{nm}\left(\varepsilon_{\max } / 10^{4} \mathrm{M}^{-1} \mathrm{~cm}^{-1}\right)$

$\mathbf{R u}(\boldsymbol{\mu}$-1)Ru 288 (16.0), 338 (4.2), 458 (4.1)

Os $\left(\boldsymbol{\mu}\right.$-1)Os $291(14.7), 374(3.6), 449(3.2), 620^{b}(0.80), 825^{b}(0.072)$

$\mathbf{R u}(\boldsymbol{\mu}-\mathbf{2}) \mathbf{R u} 287$ (14.4), 339 (4.1), 458 (3.6)

$\operatorname{Ru}\left(\boldsymbol{\mu}\right.$-2)Os 288 (14.6), 359 (4.5), $461(3.7), 620^{b}(0.36)$

${ }^{a}$ In MeCN, at $293 \mathrm{~K} .{ }^{b}$ Estimated center position of an unresolved broad absorption band, not a real absorption maximum.

ligand (bpy) charge-transfer $\left({ }^{1} \mathrm{MLCT}\right)$ transitions, in analogy with the reference complexes $\left[\mathrm{Ru}(\mathrm{bpy})_{3}\right]^{2+},{ }^{37}[\mathrm{Os}(\mathrm{b}-$ py) $\left.{ }_{3}\right]^{2+38-40}$ and $\left[\operatorname{Ru}(i p)_{3}\right]^{2+}$ (ip = imidazo[4,5-f][1,10]phenanthroline $).^{41}$ For the Os-containing compounds Os( $\boldsymbol{\mu}$ 1)Os and $\mathbf{R u}(\boldsymbol{\mu}-2)$ Os an additional weak and broad band is observed in the 550-700 $\mathrm{nm}$ region (see Figure 2), assigned to ${ }^{3}$ MLCT transitions partially allowed as a result of the heavy-atom effect, typical for $\left[\mathrm{Os}(\mathrm{bpy})_{3}\right]^{2+}$-type complexes. ${ }^{38-40}$ The origin of the very weak absorption of complex Os( $\boldsymbol{\mu - 1})$ Os between $750-900 \mathrm{~nm}$ is unclear. The bands around 290 are assigned to bpy- and/or ip-centered transitions $\left({ }^{1} \mathrm{IL}_{\mathrm{bpy}}{ }^{1} \mathrm{IL}_{\mathrm{ip}}\right)$. The absorption bands due to these intraligand transitions have very similar shapes and absorption maxima and cannot be distinguished in the spectra. ${ }^{37,41}$ The composed absorption band in the $320-410 \mathrm{~nm}$ region can be attributed to the spiropyran subunit $\left({ }^{1} \mathrm{IL}_{\mathrm{Sp}}\right)$. Since the metal $(\alpha$-diimine $)$ moieties absorb only weakly in this region, we can address the spiropyran part of the system fairly selectively by exciting into the ${ }^{1} \mathrm{IL}_{\mathrm{Sp}}$ transition. On the other hand, the metal $(\alpha$-diimine $)$ chromophores can be excited selectively by irradiating into the lowest ${ }^{1}$ MLCT band.

Photoreactivity. Excitation of a closed spiropyran species with light normally results in its photochemical conversion

(37) Juris, A.; Balzani, V.; Barigelletti, F.; Campagna, S.; Belser, P.; von Zelewsky, A. Coord. Chem. Rev. 1988, 84, 85-277.

(38) Demas, J. N.; Crosby, G. A. J. Am. Chem. Soc. 1971, 93, 2841-2847.

(39) Fujita, I.; Kobayashi, H. Z. Phys. Chem. 1972, 79, 309-314.

(40) Decurtins, S.; Felix, F.; Ferguson, J.; Güdel, H. U.; Ludi, A. J. Am. Chem. Soc. 1980, 102, 4102-4106.

(41) Wu, J. Z.; Ji, L. N. Transition Met. Chem. 1999, 24, 299-303.

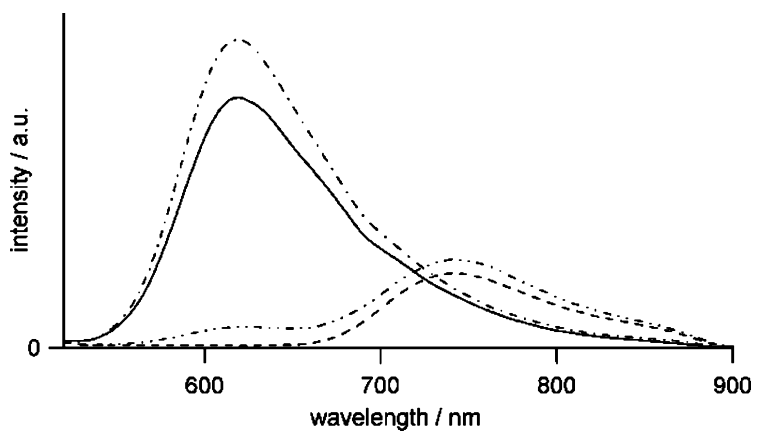

Figure 3. Emission spectra of $\mathbf{R u}(\boldsymbol{\mu}-\mathbf{1}) \mathbf{R u}$ (solid line), Os( $\boldsymbol{\mu}-\mathbf{1}) \mathrm{Os}$ (dashed line), $\mathbf{R u}(\boldsymbol{\mu}-\mathbf{2}) \mathbf{R u}$ (dashed-single-dotted line) and $\mathbf{R u}(\boldsymbol{\mu}-\mathbf{2}) \mathbf{O s}$ (dasheddouble-dotted line) in air-equilibrated $\mathrm{MeCN}$ at $293 \mathrm{~K} ; \lambda_{\text {exc }}=450 \mathrm{~nm}$.

to the open merocyanine form, which is characterized by the rise of a new strong absorption band in the 500-600 $\mathrm{nm}$ spectral region. ${ }^{11}$ For monosubstituted nitrospiropyrans Ru-3c and Os-3c (see Chart 1), the photochemical formation of the corresponding open-form structures Ru-3o and Os3o was indeed well observed, but the efficiency of this process is low as a result of energy transfer from the excited spiropyran moiety to the metal center. ${ }^{16}$ No changes, however, were observed in the electronic absorption spectra of investigated dinuclear complexes $\mathbf{R u}(\boldsymbol{\mu}-\mathbf{1}) \mathbf{R u}, \mathbf{O s}(\boldsymbol{\mu}-\mathbf{1}) \mathbf{O s}$, $\mathbf{R u}(\boldsymbol{\mu}-\mathbf{2}) \mathbf{R u}$, and $\mathbf{R u}(\boldsymbol{\mu}-\mathbf{2}) \mathbf{O s}$ under the same experimental conditions, not even after prolonged irradiation into the ${ }^{1} \mathrm{IL}_{\mathrm{Sp}}$ band with $334 \mathrm{~nm}$ light at $223 \mathrm{~K}$. From these results it is concluded that $\operatorname{Ru}(\mu-1) \mathbf{R u}, \mathbf{O s}(\mu-1) \mathbf{O s}, \operatorname{Ru}(\mu-2) \mathbf{R u}$, and $\mathbf{R u}(\boldsymbol{\mu}$-2)Os are not photochromic.

There are two possible explanations for the fact that the ring-opening reaction, reported for the monosubstituted nitrospiropyrans, is completely inhibited in the dinuclear systems. First, because of the presence of a second energy acceptor subunit, the energy transfer may become so fast that the photochromic reaction can no longer compete. Second, it is possible that the substitution of the spiropyran nitro group in the mononuclear complexes with the additional ruthenium or osmium center has changed the intrinsic properties of the spiropyran, so that the ring-opening pathway is no longer available. These options will be discussed hereinafter.

Luminescence Properties. Emission spectra of complexes $\operatorname{Ru}(\mu-1) \mathbf{R u}, \operatorname{Os}(\mu-1) O s, \mathbf{R u}(\mu-2) \mathbf{R u}$, and $\mathbf{R u}(\mu-2)$ Os in airequilibrated acetonitrile $(\mathrm{MeCN})$ are shown in Figure 3. Emission maxima, quantum yields, and lifetimes of all investigated complexes and selected reference materials are reported in Table 2.

Diruthenium complexes $\mathbf{R u}(\boldsymbol{\mu}-\mathbf{- 1}) \mathbf{R u}$ and $\mathbf{R u}(\boldsymbol{\mu}-\mathbf{2}) \mathbf{R u}$ emit at room temperature at 619 and $618 \mathrm{~nm}$, respectively. The shape and intensity of the emission bands, as well as the emission lifetimes (see Table 2), reveal their origin in the Ru(bpy)-localized ${ }^{3}$ MLCT excited state. ${ }^{37}$ Diosmium complex Os( $\boldsymbol{\mu}$-1)Os emits at $741 \mathrm{~nm}$. The emission clearly originates from an Os-to-bpy ${ }^{3} \mathrm{MLCT}$ state. ${ }^{43}$

(42) Nakamaru, K. Bull. Chem. Soc. Jpn. 1982, 55, 1639-1640.

(43) Kober, E. M.; Caspar, J. V.; Lumpkin, R. S.; Meyer, T. J. J. Phys. Chem. 1986, 90, 3722-3734.

(44) Jing, B. W.; Zhang, M. H.; Shen, T. Spectrochim. Acta A 2004, 60, $2635-2641$. 
Table 2. Emission Maxima $\left(\lambda_{\max }\right)$, Lifetimes $(\tau)$, and Quantum Yields $\left(\varphi_{\mathrm{em}}\right)$ of the Investigated Complexes $\mathbf{R u}(\boldsymbol{\mu}-\mathbf{1}) \mathbf{R u}, \mathbf{O s}(\boldsymbol{\mu}-\mathbf{1}) \mathbf{O s}, \mathbf{R u}(\boldsymbol{\mu}-\mathbf{2}) \mathbf{R u}$, and $\mathbf{R u}(\boldsymbol{\mu}-2) \mathbf{O s}$ and Reference Compounds ${ }^{a}$

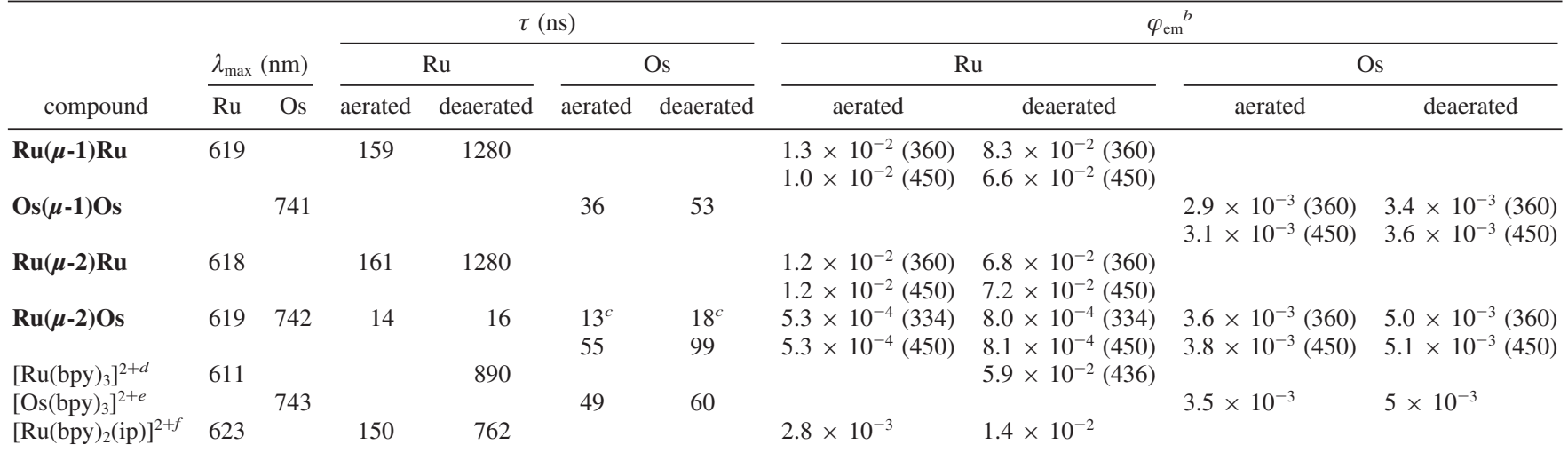

${ }^{a}$ Conditions: MeCN, 293 K. ${ }^{b}$ Excitation wavelength (nm) in brackets. ${ }^{c}$ Growth of the Os emission by energy transfer from the excited Ru center. ${ }^{d}$ Ref 42. ${ }^{e}$ Refs.22, $43{ }^{f} \operatorname{Ref} 44$; ip = imidazo[ $[4,5-f][1,10]$ phenanthroline.

Mixed-metal complex $\mathbf{R u}(\boldsymbol{\mu}$-2)Os exhibits both $\mathrm{Ru}(\mathrm{bpy})$ and Os(bpy) centered emissions at 619 and $742 \mathrm{~nm}$, respectively. The ruthenium emission is strongly quenched, as a result of an energy transfer to the osmium center. From the corresponding emission lifetimes (Table 2) it can be determined that the rate of this process can be expressed with $k_{\mathrm{ET}}=6.6 \times 10^{7} \mathrm{~s}^{-1}$.

The metal-metal distance in complex $\mathbf{R u}(\boldsymbol{\mu}-\mathbf{2}) \mathbf{O s}$ is $r_{\mathrm{DA}}$ $=2.3 \mathrm{~nm}$. In the literature, a number of heterodinuclear $\mathrm{Ru} /$ Os donor-bridge-acceptor systems with similar metal-metal distances have been reported, in which the bridges are well conjugated ${ }^{45-48}$ and have energy levels positioned between those of the donor ruthenium and those of the acceptor osmium centers. ${ }^{49}$ The energy transfer in these triads is 1 to 3 orders of magnitude faster than we have found for complex $\mathbf{R u}(\boldsymbol{\mu}-2)$ Os. On the other hand, for similarly spaced Ru and Os termini separated by non-conjugated bridges, energy transfer rates are comparable ${ }^{50-53}$ to that determined for complex $\mathbf{R u}(\boldsymbol{\mu}-\mathbf{2}) \mathbf{O s}$, or even 1 order of magnitude lower. ${ }^{54}$ From this comparison it may be concluded that, as expected, the closed spiropyran is a fairly good insulator for the $\mathrm{Ru}$ to-Os energy transfer.

To ascertain whether the spiropyran moiety in the D-BL-A triad acts merely as a structural motif keeping the donor and

(45) Barigelletti, F.; Flamigni, L.; Balzani, V.; Collin, J. P.; Sauvage, J. P.; Sour, A.; Constable, E. C.; Thompson, A. J. Am. Chem. Soc. 1994, $116,7692-7699$.

(46) Grosshenny, V.; Harriman, A.; Hissler, M.; Ziessel, R. J. Chem. Soc., Faraday Trans. 1996, 92, 2223-2238.

(47) Schlicke, B.; Belser, P.; De Cola, L.; Sabbioni, E.; Balzani, V. J. Am. Chem. Soc. 1999, 121, 4207-4214.

(48) Welter, S.; Salluce, N.; Belser, P.; Groeneveld, M.; De Cola, L. Coord. Chem. Rev. 2005, 249, 1360-1371.

(49) El-Ghayoury, A.; Harriman, A.; Ziessel, R. J. Phys. Chem. A 2000, 104, 7906-7915.

(50) Furue, M.; Yoshidzumi, T.; Kinoshita, S.; Kushida, T.; Nozakura, S.; Kamachi, M. Bull. Chem. Soc. Jpn. 1991, 64, 1632-1640.

(51) De Cola, L.; Balzani, V.; Barigelletti, F.; Flamigni, L.; Belser, P.; von Zelewsky, A.; Frank, M.; Vögtle, F. Inorg. Chem. 1993, 32, 5228 5238 .

(52) Vögtle, F.; Frank, M.; Nieger, M.; Belser, P.; von Zelewsky, A.; Balzani, V.; Barigelletti, F.; De Cola, L.; Flamigni, L. Angew. Chem., Int. Ed. 1993, 32, 1643-1646.

(53) Balzani, V.; Barigelletti, F.; Belser, P.; Bernhard, S.; De Cola, L.; Flamigni, L. J. Phys. Chem. 1996, 100, 16786-16788.

(54) Hammarström, L.; Barigelletti, F.; Flamigni, L.; Armaroli, N.; Sour, A.; Collin, J. P.; Sauvage, J. P. J. Am. Chem. Soc. 1996, 118, 1197211973.

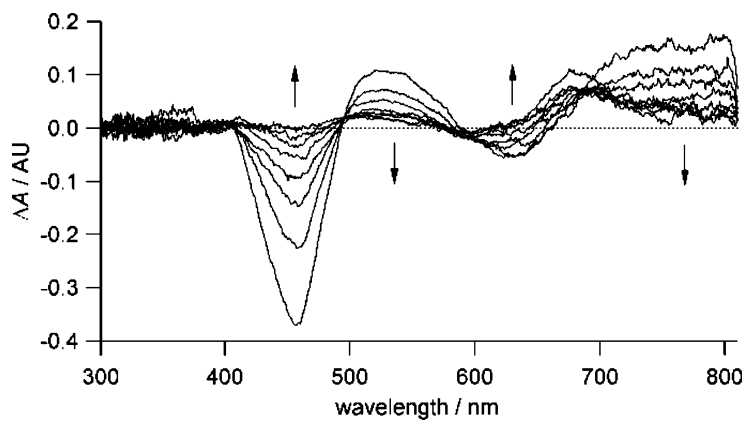

Figure 4. Transient absorption difference spectrum of $\mathbf{R u}(\boldsymbol{\mu}-\mathbf{2}) \mathbf{R u}$ in airequilibrated $\mathrm{MeCN}$ at $293 \mathrm{~K}$, with $60 \mathrm{~ns}$ intervals between frames; $\lambda_{\mathrm{exc}}=$ $450 \mathrm{~nm}$.

acceptor chromophores together at a fixed distance and the electronic energy transfer occurs through space without any bridge mediation, the value of $k_{\mathrm{ET}}$ was calculated according to the classical Förster formula for isotropic conditions.

$$
k_{\mathrm{ET}}=5.87 \times 10^{-25}\left(\Phi_{\mathrm{D}} / n^{4} \tau_{\mathrm{D}} r_{\mathrm{DA}}\right) \int_{0}^{\infty} F_{\mathrm{D}}(\tilde{v}) \varepsilon_{\mathrm{A}}(\tilde{v}) \mathrm{d} \tilde{v} / \tilde{v}^{4}
$$

For the $\mathbf{R u}\left(\boldsymbol{\mu}\right.$-2)Os complex, $\Phi_{\mathrm{D}}=0.072$ (emission quantum yield of $\mathbf{R u}(\boldsymbol{\mu}-\mathbf{2}) \mathbf{R u}$ ), $\tau_{\mathrm{D}}=1.28 \mu$ s (emission lifetime of $\mathbf{R u}(\boldsymbol{\mu}$-2)Ru$), r_{\mathrm{DA}}=2.3 \mathrm{~nm}, n=1.3442$ (refractive index of acetonitrile) and the value of the overlap integral is $4.38 \times 10^{-14} \mathrm{M}^{-1} \mathrm{~cm}^{-1}$ (calculated using the Mathcad 14.0 software).

The value $k_{\mathrm{ET}}=2.98 \times 10^{6} \mathrm{~s}^{-1}$ obtained for $\mathbf{R u}(\boldsymbol{\mu}-\mathbf{2}) \mathbf{O s}$ from eq 1 is smaller by more than 1 order of magnitude than the experimental value of $6.6 \times 10^{7} \mathrm{~s}^{-1}$. This result reveals that the dipole-dipole Förster mechanism itself cannot account for the observed energy-transfer process in $\mathbf{R u}(\boldsymbol{\mu}-\mathbf{2}) \mathbf{O s}$ and a superexchange mechanism plays a major role, in line with Figure 1 (left). Indeed, it would be very interesting to compare the energy-transfer mechanism for the open merocyanine-containing bridge if this heterodinuclear triad were photochromic.

Nanosecond Transient Absorption Spectroscopy. Transient UV-vis absorption (TA) spectra of diruthenium systems $\mathbf{R u}(\boldsymbol{\mu}-\mathbf{1}) \mathbf{R u}$ and $\mathbf{R u}(\boldsymbol{\mu}-\mathbf{2}) \mathbf{R u}$ with ns time resolution (air-equilibrated $\mathrm{MeCN}, 293 \mathrm{~K}$; see Figure 4) and the corresponding excited-state lifetimes $\tau=128$ and $120 \mathrm{~ns}$, 


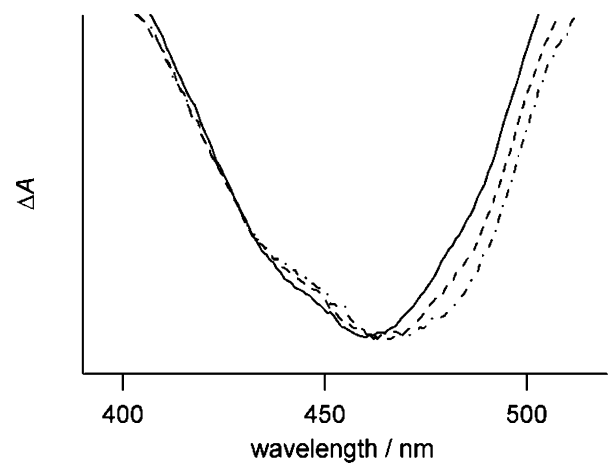

Figure 5. Details of the transient absorption difference spectrum of $\mathbf{R u}(\boldsymbol{\mu}$ 2)Os in air-equilibrated $\mathrm{MeCN}$ at $293 \mathrm{~K}$, showing the ${ }^{3} \mathrm{MLCT}$ bleach at 3 ns (solid line), 12 ns (dashed line) and $21 \mathrm{~ns}$ (dashed-dotted line) after laser excitation at $450 \mathrm{~nm}$. Absorptions are normalized for clarity.

respectively, are characteristic of ruthenium-to-diimine ${ }^{3}$ MLCT excited states of complexes like $\left[\mathrm{Ru}(\mathrm{bpy})_{3}\right]^{2+}{ }^{25}$ The negative feature around $630 \mathrm{~nm}$ is caused by the emission of the complex. The recorded spectra are independent of whether the system is excited into the ${ }^{1} \mathrm{IL}_{\mathrm{Sp}}$ band with 334 $\mathrm{nm}$ light, or into the ${ }^{1} \mathrm{MLCT}$ band with $450 \mathrm{~nm}$ light. After the decay of the excited-state species, an absorption feature around $680 \mathrm{~nm}$ remains permanently. The same phenomenon was observed for Ru-3c. ${ }^{16}$ Its origin is unclear.

The spectral shape of the transient absorption spectrum observed for diosmium complex $\mathbf{O s}(\boldsymbol{\mu}-\mathbf{1}) \mathbf{O}$ s and the corresponding excited-state lifetime $\tau=40$ ns (air-equilibrated MeCN, $293 \mathrm{~K}$ ) reveal optical population of the osmium-tobpy ${ }^{3}$ MLCT excited state. ${ }^{56}$ Also in this case, the transient is independent of the excitation wavelength.

Excitation of mixed-metal complex $\mathbf{R u}(\boldsymbol{\mu}$-2)Os with 450 $\mathrm{mm}$ light initially populates both the ruthenium and osmium ${ }^{3}$ MLCT states. The development of the TA spectrum in time points to energy transfer from the ruthenium to osmium chromophore. The latter process is apparent from the detail of the MLCT bleach showing a gradual shift to longer wavelengths in time (see Figure 5) and from the excitedstate life $\tau=69 \mathrm{~ns}$ (air-equilibrated $\mathrm{MeCN}, 293 \mathrm{~K}$ ) that is much closer to that of $\mathbf{O s}(\boldsymbol{\mu}-\mathbf{1}) \mathbf{O s}$ than $\mathbf{R u}(\boldsymbol{\mu}-\mathbf{1}) \mathbf{R u}$ or $\mathbf{R u}(\boldsymbol{\mu}$ 2)Ru (see above).

The excited-state lifetimes determined from the ns TA spectra correspond to the emission lifetimes in Table 2.

Picosecond Time-Resolved Transient Absorption Spectroscopy. Transient absorption spectra of complexes $\mathbf{R u}(\boldsymbol{\mu}-\mathbf{1}) \mathbf{R u}, \mathbf{R u}(\boldsymbol{\mu}-\mathbf{2}) \mathbf{R u}$, and $\mathbf{R u}(\boldsymbol{\mu}-\mathbf{2}) \mathbf{O s}$ in $\mathrm{MeCN}$ were recorded in the picoseconds time domain in an attempt to determine the rate of energy transfer to the metal center upon excitation of the spiropyran part of the investigated molecules. Optical population of the ${ }^{1} \mathrm{IL}_{\mathrm{Sp}}$ excited-state was achieved by laser excitation with $360 \mathrm{~nm}$ light. We monitored the time development of the transient absorption spectra, having focused on the MLCT bleach. Its initial intensity should increase if an energy transfer from the spiropyran moiety to the acceptor metal center were to take place in the investigated time domain, which would allow the

(55) Miedlar, K.; Das, P. K. J. Am. Chem. Soc. 1982, 104, 7462-7469.

(56) Creutz, C.; Chou, M.; Netzel, T. L.; Okumura, M.; Sutin, N. J. Am. Chem. Soc. 1980, 102, 1309-1318. determination of the corresponding energy transfer rate. However, from the first frame on, only the pattern characteristic of ruthenium- and/or osmium-to-bpy ${ }^{3}$ MLCT excited states was recorded. No clear growth in the bleach amplitude was observed for any of the complexes, indicating that the bridge-to-metal acceptor energy transfer is faster than the time resolution of our apparatus and must therefore be completed on a subpicosecond time scale.

For comparison, we also recorded picosecond transient absorption spectra of monosubstituted nitrospiropyrans Ru$\mathbf{3 c}$ and Os-3c. Also for these systems the energy transfer is too fast to be observed.

As the energy transfer from the spiropyran moiety to the acceptor metal center is faster than the ps time resolution of our apparatus for both the monometallic and the bimetallic complexes, a comparison between the rates cannot be made. This makes it difficult to prove directly whether the photostability of complexes $\mathbf{R u}(\boldsymbol{\mu}-\mathbf{1}) \mathbf{R u}, \mathbf{O s}(\boldsymbol{\mu}-\mathbf{1}) \mathbf{O s}, \mathbf{R u}(\boldsymbol{\mu}-\mathbf{2}) \mathbf{R u}$, and $\mathbf{R u}(\boldsymbol{\mu}-\mathbf{2}) \mathbf{O s}$ is due to the critically reduced $\mathrm{IL}_{\mathrm{Sp}}$ lifetime. However, considering the fact that the $\mathrm{IL}_{\mathrm{Sp}} \rightarrow$ MLCT energy transfer is already extremely fast in $\mathbf{R u - 3 c}$ and $\mathbf{O s - 3 c}$, and yet the photochromic reaction still occurs, ${ }^{16}$ it appears unlikely to become so fast in the dinuclear systems that the ring-opening quantum yield is essentially reduced to zero. Therefore, the lack of photoreactivity of the bimetallic systems is more likely the result of changed excited-state properties of the spiropyran subunit. The substitution of the nitro group with the second metal coordination center makes the ring-opening pathway unavailable.

Voltammetry. The redox potentials of all investigated bimetallic complexes and selected reference compounds are reported in Table 3. DMF was used owing to the good solubility of the initial reduction products in this solvent, in contrast to less polar MeCN or PrCN.

For both $\mathbf{R u}(\boldsymbol{\mu}-\mathbf{1}) \mathbf{R u}$ (see Figure 6) and $\mathbf{O s}(\boldsymbol{\mu}-\mathbf{1}) \mathbf{O s}$, three chemically reversible cathodic waves can be distinguished, each corresponding to a transfer of two electrons. By analogy with the reduction of $\left[\mathrm{Ru}(\mathrm{bpy})_{3}\right]^{2+57}$ and $\left[\mathrm{Os}(\mathrm{bpy})_{3}\right]^{2+},{ }^{28}$ respectively, these three waves are assigned to the paired sequential reductions of the 2,2'-bipyridine ligands at the metal centers to corresponding radical anions. Both $\mathbf{R u}(\boldsymbol{\mu}$ 1)Ru and $\mathbf{O s}(\boldsymbol{\mu}-\mathbf{1}) \mathbf{O}$ also exhibit a single two-electron anodic wave (see Table 3) that can be assigned to the simultaneous oxidation of the two ruthenium(II) and osmium(II) centers, respectively. The absence of resolved oneelectron waves proves that the spiropyran-bridged metal centers do not interact electronically in the ground state.

For complexes $\mathbf{R u}(\boldsymbol{\mu}-\mathbf{2}) \mathbf{R u}$ and $\mathbf{R u}(\boldsymbol{\mu}-\mathbf{2}) \mathbf{O s}$ two partly overlapping one-electron cathodic waves were observed in the cyclic voltammograms, followed by two unresolved twoelectron waves. The second one-electron and both twoelectron waves are clearly chemically reversible in both cases. However, for the first one-electron wave no clear counter peak was recorded on the reverse anodic scan. This observation points to some chemical instability of the primary

(57) Sutin, N.; Creutz, C. Adv. Chem. Ser. 1978, 168, 1-27.

(58) Saji, T.; Aoyagui, S. J. Electroanal. Chem. 1975, 58, 401-410.

(59) Saji, T.; Aoyagui, S. J. Electroanal. Chem. 1975, 1-10. 
Table 3. Redox Potentials of Investigated Complexes $\mathbf{R u}(\boldsymbol{\mu}-\mathbf{1}) \mathbf{R u}, \mathbf{O s}(\boldsymbol{\mu}-\mathbf{1}) \mathbf{O s}, \mathbf{R u}(\boldsymbol{\mu}-\mathbf{2}) \mathbf{R u}$, and $\mathbf{R u}(\boldsymbol{\mu}-\mathbf{2}) \mathbf{O s},{ }^{a}$ and Reference Compounds

\begin{tabular}{|c|c|c|c|c|c|c|c|c|}
\hline compound & solvent & $E_{1 / 2} / \mathrm{V} \mathrm{Ru}{ }^{\mathrm{II} / \mathrm{III}}$ & $E_{1 / 2} / \mathrm{V} \mathrm{Os}{ }^{\mathrm{II} / I I I}$ & $E_{1 / 2} / \mathrm{V} \mathrm{ip}{ }^{0 /-}$ & $E_{1 / 2} / \mathrm{V}\left(\mathrm{bpy}^{0 /-}\right)_{\mathrm{I}}$ & $E_{1 / 2} / \mathrm{V}\left(\mathrm{bpy}^{0 /-}\right)_{\mathrm{II}}$ & $E_{1 / 2} / \mathrm{V}\left(\mathrm{bpy}^{0 /-}\right)_{\mathrm{III}}$ & $\Delta E_{\mathrm{p}} / \mathrm{V} \mathrm{Fc} / \mathrm{Fc}^{+}$ \\
\hline $\mathbf{R u}(\mu-1) \mathbf{R u}$ & DMF & $+0.80(0.08)$ & & & $-1.74(0.06)$ & $-1.93(0.06)$ & $-2.19(0.07)$ & 0.07 \\
\hline Os $(\mu-1) O s$ & DMF & & $+0.35(0.09)$ & & $-1.66(0.07)$ & $-1.84(0.06)$ & $-2.19(0.07)$ & 0.07 \\
\hline $\mathbf{R u}(\mu-2) \mathbf{R u}$ & DMF & $+0.83,+0.77^{b, c}$ & & $-1.67^{c, d}$ & $-1.74(0.06)$ & $-1.95(0.08)$ & $-2.21(0.10)$ & 0.09 \\
\hline$\left[\mathrm{Ru}(\mathrm{bpy})_{3}\right]^{2+e}$ & $\mathrm{MeCN}$ & +0.89 & & & -1.72 & -1.93 & -2.18 & \\
\hline$\left[\mathrm{Os}(\mathrm{bpy})_{3}\right]^{2+f}$ & DMF & & +0.35 & & -1.67 & -1.85 & -2.17 & \\
\hline
\end{tabular}

${ }^{a}$ Redox potentials in V vs ferrocene/ferrocenium, determined from cyclic and Osteryoung square-wave voltammetric scans. Conditions: $T=293 \mathrm{~K}$, $\mathrm{Pt}$ microdisk working electrode, $v=100 \mathrm{mV} \mathrm{s}^{-1}$ (cyclic voltammetry), $f=30 \mathrm{~Hz}$ ( $\mathrm{s}-\mathrm{W}$ voltammetry). $\Delta E_{\mathrm{p}}$ values in $\mathrm{V}$ (cyclic voltammetry) are given in the brackets. ${ }^{b}$ Value taken from the square-wave voltammetric scan. ${ }^{c}$ Unresolved in the cyclic voltammogram. ${ }^{d}$ No clear anodic counter-peak observed in the cyclic voltammogram. ${ }^{e}$ Ref $57 .{ }^{f}$ Refs $58,59$.

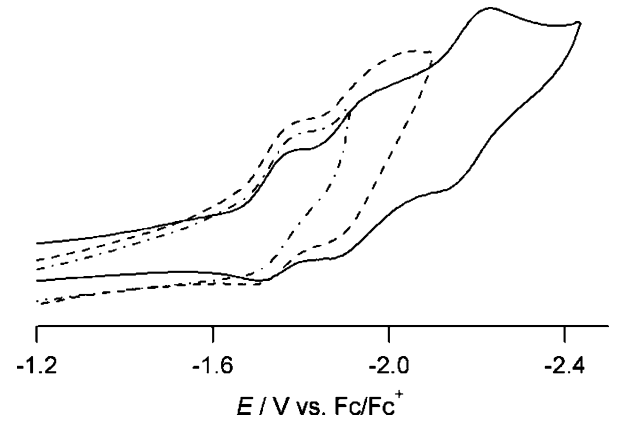

Figure 6. Cathodic region of the cyclic voltammogram of complex $\mathbf{R u}(\boldsymbol{\mu}$ 1)Ru showing the sequence of three bpy-localized bielectronic waves. Conditions: $\mathrm{DMF} / 10^{-3} \mathrm{M} \mathrm{Bu}_{4} \mathrm{NPF}_{6}, T=293 \mathrm{~K}$, Pt microdisk working electrode, $v=100 \mathrm{mV} \mathrm{s}^{-1}$.

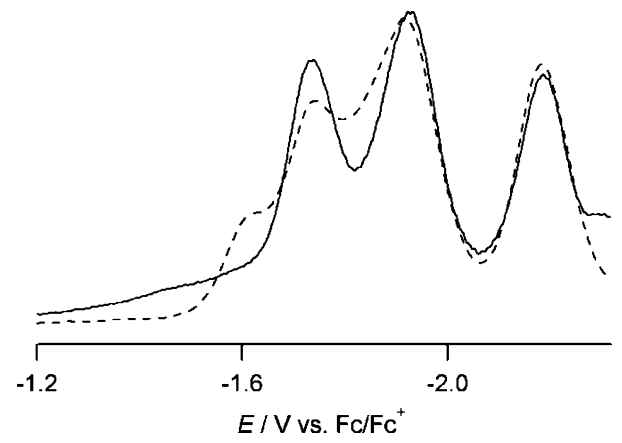

Figure 7. Cathodic square-wave voltammetric scan of $\mathbf{R u}(\boldsymbol{\mu}-\mathbf{1}) \mathbf{R u}$ (solid line) and $\mathbf{R u}\left(\boldsymbol{\mu}\right.$-2)Os (dashed line). Conditions: DMF $/ 10^{-3} \mathrm{M} \mathrm{Bu}_{4} \mathrm{NPF}_{6}, T$ $=293 \mathrm{~K}, \mathrm{Pt}$ microdisk working electrode, $f=30 \mathrm{~Hz}$ (corresponding to the same time domain as in Figure 6).

reduction product, in agreement with the corresponding spectroelectrochemical investigations (see below). The difference in the cathodic responses of complexes $\mathbf{R u}(\boldsymbol{\mu}-\mathbf{1}) \mathbf{R u}$ and $\mathbf{O s}(\mu-1) O s$ on one hand and $\mathbf{R u}(\mu-2) \mathbf{R u}$ and $\mathbf{R u}(\mu-2)$ Os on the other is most apparent from cathodic branches of the corresponding square-wave voltammograms (see Figure 7). A comparison of the reduction potentials of $\mathbf{R u}(\boldsymbol{\mu}-\mathbf{2}) \mathbf{R u}$ and $\mathbf{R u}\left(\boldsymbol{\mu}\right.$-2)Os with the reference $\left[\mathrm{Ru}(\mathrm{bpy})_{3}\right]^{2+57}$ and complexes $\mathbf{R u}(\boldsymbol{\mu}-\mathbf{1}) \mathbf{R u}$ and $\mathbf{O s}(\boldsymbol{\mu}-\mathbf{1})$ Os allows the assignment of the second (chemically reversible) one-electron wave of $\mathbf{R u}(\boldsymbol{\mu}$ 2) $\mathbf{R u}$ and $\mathbf{R u}(\boldsymbol{\mu}-\mathbf{2}) \mathbf{O s}$ to the reduction of the spiropyranbound bpy ligand chelating the $\left[\mathrm{Ru}(\mathrm{bpy})_{3}\right]$ metal center. The first (chemically irreversible) cathodic step can thus be attributed in both cases to the reduction of the spiropyranbound imidazophenanthroline (ip) ligand. The two bielectronic cathodic waves of $\mathbf{R u}(\boldsymbol{\mu}-\mathbf{2}) \mathbf{R u}$ and $\mathbf{R u}(\boldsymbol{\mu}-\mathbf{2}) \mathbf{O s}$ are assigned to the sequential reductions of the ancillary bpy ligands, similar to complexes $\mathbf{R u}(\boldsymbol{\mu}-\mathbf{1}) \mathbf{R u}$ and $\mathbf{O s}(\boldsymbol{\mu}-\mathbf{1}) \mathbf{O}$.

For complex $\mathbf{R u}(\boldsymbol{\mu}-\mathbf{2}) \mathbf{R u}$ a broad reversible anodic wave

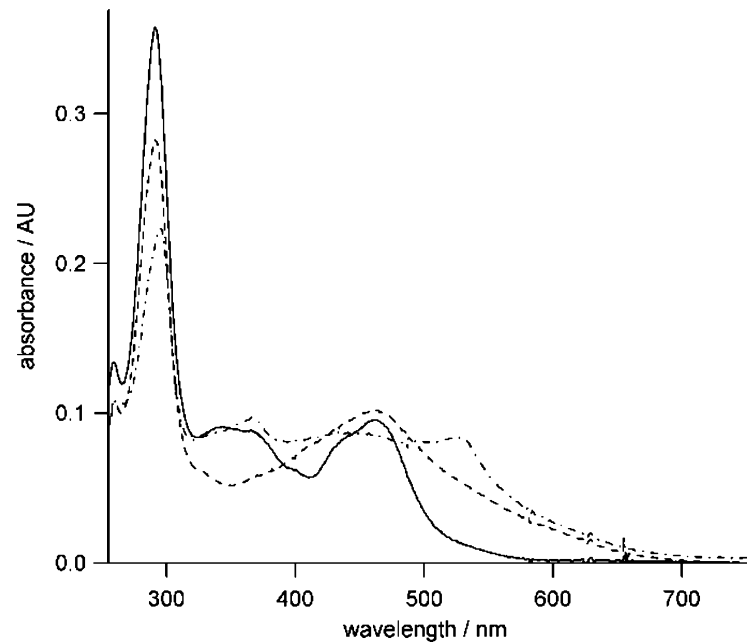

Figure 8. UV-vis absorption spectra of $\mathbf{R u}(\boldsymbol{\mu}-\mathbf{1}) \mathbf{R u}$ (solid line) and its reduction products in DMF at $293 \mathrm{~K}$ recorded in an OTTLE cell halfway through (dashed line), and at the end (dashed-dotted line) of the first twoelectron cathodic wave (Figure 6 and Table 3).

appeared in the cyclic voltammogram. The corresponding square-wave voltammogram discerned two overlapping anodic waves, in agreement with the different coordination spheres of the ruthenium(II) centers in $\mathbf{R u}(\boldsymbol{\mu}-\mathbf{2}) \mathbf{R u}$. Their exact assignment to a particular ruthenium center was not attempted.

The cyclic voltammogram of complex $\operatorname{Ru}(\boldsymbol{\mu}$-2)Os shows two well-separated chemically reversible anodic waves at +0.39 and $+0.78 \mathrm{~V}$ belonging with no doubts to one-electron oxidations of the osmium(II) and ruthenium(II) sites, respectively.

Spectroelectrochemistry. When scanning through the first two-electron cathodic wave of complex $\mathbf{R u}(\boldsymbol{\mu}-\mathbf{1}) \mathbf{R u}$ in a thinlayer spectroelectrochemical cell, two different processes can be distinguished by the corresponding UV-vis spectral changes (see Figure 8). Initially, the intensities of both the ${ }^{1} \mathrm{IL}_{\mathrm{bpy}}$ and ${ }^{1} \mathrm{IL}_{\mathrm{Sp}}$ absorption decrease, which is accompanied by the appearance of a broad absorption band at a longer wavelength. Half-way over the first cathodic peak, this process is completed. When the scan is continued beyond the first cathodic peak, a different spectral change is observed, which closely resembles that recorded during the initial $1 \mathrm{e}$ reduction of $\left[\mathrm{Ru}(\mathrm{bpy})_{3}\right]^{2+}{ }^{60}$ Further UV-vis spectral changes show that the following two bielectronic cathodic steps are localized on the two ancillary bpy ligands of the $\left[\mathrm{Ru}(\mathrm{bpy})_{3}\right]^{2+}$ substituents. ${ }^{60}$ These reductions are completely reversible,

(60) Heath, G. A.; Yellowlees, L. J.; Braterman, P. S. Chem. Commun. 1981, 287-289. 


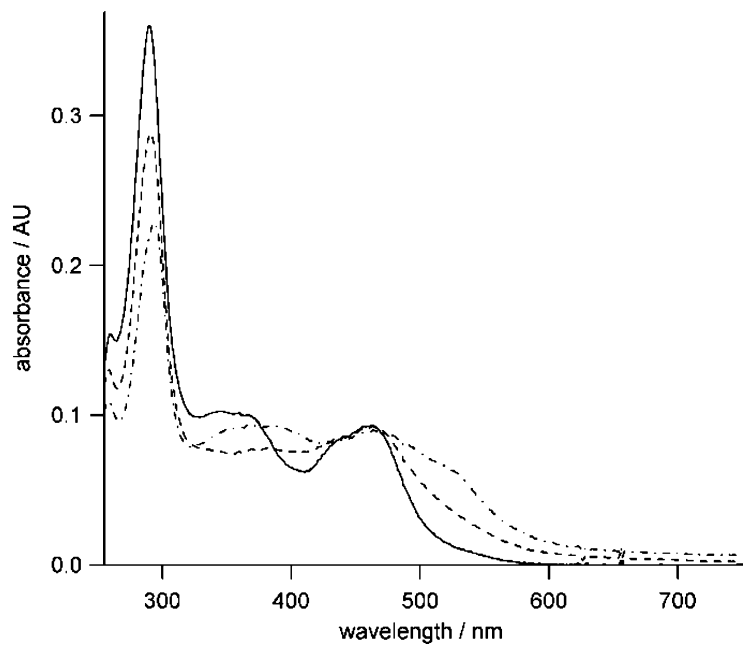

Figure 9. UV-vis absorption spectra of $\mathbf{R u}(\boldsymbol{\mu}-\mathbf{2}) \mathbf{R u}$ (solid line) and its reduction products recorded in DMF at $293 \mathrm{~K}$ in an OTTLE cell at electrode potentials beyond the first (dashed line), and second (dashed-dotted line) one-electron cathodic waves (Figure 7 and Table 3).

which also applies for the second process within the first cathodic wave of $\mathbf{R u}(\boldsymbol{\mu}-\mathbf{1}) \mathbf{R u}$. In contrast, the initial reduction of $\mathbf{R u}(\boldsymbol{\mu}-\mathbf{1}) \mathbf{R u}$ is irreversible, as documented by the absence of an anodic counter-peak and the appearance of a new anodic wave on the reverse thin-layer cyclic voltammetric scan at a more than $1 \mathrm{~V}$ more positive potential than the original cathodic wave. Oxidation at the new anodic wave results in the recovery of the starting material, although a slight change in the shape of the ${ }^{1} \mathrm{IL}_{\mathrm{Sp}}$ band could be observed in the UV-vis spectrum, indicating that some decomposition took place as a result of the reduction/reoxidation processes.

Complex $\mathbf{O s}(\boldsymbol{\mu}-\mathbf{1}) \mathbf{O s}$ exhibits the same redox behavior as $\mathbf{R u}(\boldsymbol{\mu}$-1)Ru. For complexes $\mathbf{R u}(\boldsymbol{\mu}-\mathbf{2}) \mathbf{R u}$ (see Figure 9) and $\mathbf{R u}(\mu-2)$ Os the first one-electron reduction results in spectral changes very similar to those observed for $\mathbf{R u}(\boldsymbol{\mu}-\mathbf{1}) \mathbf{R u}$ and Os( $(\mu-1) O s$, despite the fact that in this case the first reduction is localized at a different type of bridging ligand. The UV-vis spectral changes accompanying the consecutive cathodic processes $(1 \mathrm{e}, 2 \mathrm{e}, 2 \mathrm{e})$ allow their assignment to the reductions of the bpy ligands. ${ }^{60}$ Identical to $\mathbf{R u}(\boldsymbol{\mu}-\mathbf{- 1}) \mathbf{R u}$ and Os $(\mu-1) O s$, the latter three reductions are completely reversible, whereas the initial one-electron-reduced species is reoxidized more than $1 \mathrm{~V}$ more positively with respect to the original reduction potential. The reoxidation resulted in nearly complete recovery of the starting material.

The very similar UV-vis spectral changes recorded in the course of the initial irreversible one-electron reductions of complexes $\operatorname{Ru}(\mu-1) \mathbf{R u}, \mathbf{O s}(\mu-1) O s, \operatorname{Ru}(\mu-2) \mathbf{R u}$, and $\operatorname{Ru}(\mu-$ 2)Os also closely resemble those observed earlier for $\mathbf{R u}-$ $\mathbf{3 c}$ and $\mathbf{O s - 3 c}$, where the first reductions are localized on the nitro group. ${ }^{16}$ Apparently, the addition of the first electron has a pronounced effect on the structure of the spiropyran moiety, which applies both for both the bimetallic spiropyran structures described in this paper, and the monometallic nitrospiropyran species studied previously. ${ }^{16}$ As the electrochemical reductions of all these complexes are not localized initially on the spiropyran moiety, they must be two-step processes, where first the substituents (the nitro group or the attached $\alpha$-diimine ligand) are reduced, which in turn induces a change in the spiropyran structure.

In the case of Ru-3c and Os-3c, reoxidation of the oneelectron reduced species was found to occur in two steps, where the second step resulted in the formation of the open merocyanine form of the molecule. ${ }^{16}$ We have tentatively proposed a "dimeric" structure for the first oxidation product, consisting of one neutral and one reduced spiropyran molecule. For complexes $\mathbf{R u}(\mu-\mathbf{1}) \mathbf{R u}, \mathbf{O s}(\mu-\mathbf{1}) \mathbf{O s}, \mathbf{R u}(\mu-2) \mathbf{R u}$, and $\mathbf{R u}(\mu$ 2)Os, however, the reoxidation occurs in a single step, and the formation of open-form molecules is not observed. There are two possible explanations for this behavior. One possibility is that the presence of the nitro group is essential for the electrochemical ring-opening process to occur. The second one is that the formation of the intermediate "dimeric" structure is required to obtain the open form molecules, and that the substitution of the spiropyran with a second bulky metal center inhibits this process.

\section{Conclusions}

Four dinuclear complexes containing metal centers connected by a spiropyran-based bridge were synthesized with the aim to control the electronic interaction between the metal centers by switching the state of the spiropyran moiety ON and OFF. Measurements on heterodinuclear (Ru/Os) complex $\mathbf{R u}(\boldsymbol{\mu}$-2)Os revealed that electronic energy transfer from the ruthenium donor to the osmium acceptor is rather slow when the spiropyran is in the closed (OFF) state, being dominated by a tunneling (superexchange) mechanism. Unfortunately, conversion of the spiropyran into the open merocyanine form, by either photochemical or electrochemical means, failed. Therefore, we could not determine the change in efficiency of the energy transfer under the same conditions, caused by the spiropyran ring opening. The change is expected to be significant because of increased bridge conjugation and a good energetic match between the donor and bridge levels for energy transfer by an exoergonic sequential ("hopping") mechanism. The photochemical pathway is most likely inhibited by the substitution of the original nitrospiropyran with the second polypyridine metal center, which causes a dramatic change in excited-state properties and the reaction trajectory of the spiropyran moiety.

The alternative electrochemical spiropyran ring-opening pathway, which was observed for nitrospiropyrans monosubstituted with a metal center, ${ }^{16}$ does not operate for the bridged bimetallic systems. The reason is either the absence of the nitro group or the presence of the new bulky metallic substituent preventing the formation of a key "dimeric" intermediate.

A possible way to regain the photochromism of the system would be to place a spacer group between the spiropyran and (one of) the metal centers. This could be quite a synthetic challenge, taking into account that the introduction of spacers between the spiropyran unit and the bipyridine moieties may have a destabilizing effect, as shown for ligands $\mathbf{8}$ and $\mathbf{9 .}$ Nevertheless, the envisaged restoration of the spiropyran photoreactivity justifies such efforts. 